University of Louisville

ThinkIR: The University of Louisville's Institutional Repository

Electronic Theses and Dissertations

8-2009

\title{
Shape analysis of the corpus callosum of autistic and normal subjects in neuroimaging.
}

Ahmed Aly Farag

University of Louisville

Follow this and additional works at: https://ir.library.louisville.edu/etd

\section{Recommended Citation}

Farag, Ahmed Aly, "Shape analysis of the corpus callosum of autistic and normal subjects in neuroimaging." (2009). Electronic Theses and Dissertations. Paper 425.

https://doi.org/10.18297/etd/425

This Master's Thesis is brought to you for free and open access by ThinkIR: The University of Louisville's Institutional Repository. It has been accepted for inclusion in Electronic Theses and Dissertations by an authorized administrator of ThinkIR: The University of Louisville's Institutional Repository. This title appears here courtesy of the author, who has retained all other copyrights. For more information, please contact thinkir@louisville.edu. 
Shape Analysis of the Corpus Callosum

of Autistic and Normal Subjects in Neuroimaging

By

Ahmed Aly Farag

B.S. ECE, University of Louisville, 2008

\author{
A Thesis \\ Submitted to the Faculty of the \\ University of Louisville \\ Speed School of Engineering \\ in Partial Fulfillment of the Requirements \\ for the Professional Degree of
}

\title{
MASTER OF ENGINEERING
}

Department of Electrical and Computer Engineering University of Louisville

Louisville, Kentucky

August 10, 2009 
Shape Analysis of the Corpus Callosum of Autistic and Normal Subjects in Neuroimaging

By

Ahmed Farag

B.S. ECE, University of Louisville, 2008

A Thesis Approved on

August 10, 2009

By the following Thesis Committee:

Dr. James Graham, Thesis Director

Dr. Aly Farag

Dr. Manuel Casanova

Dr. Mohamed Ahmed

Dr. Roger Bradshaw 


\section{DEDICATION}

To my family and friends, who have helped me develop into the person I am today, especially my parents who have dedicated their lives to their children. All praises are to Allah, God Almighty, Who is with us at all times. 


\section{ACKNOWLEDGEMENTS}

I would like to extend my gratitude to my Thesis Director Dr. James Graham, and Thesis Committee (Dr. Casanova, Dr. Bradshaw, Dr. Ahmed and Dr. Farag) for their assistance and guidance. Special thanks are to CVIP Lab members Mostafa Abdelrahman, Shireen Elhabian, and Dr. Hossam Abdelmunim for their significant assistance with this project. I acknowledge the help of Melih Aslan, Dr. Asim Ali, Ham Rara and Mike Miller at the CVIP Lab. I would also like to acknowledge with much gratitude and respect, my father, friend, and adviser, Dr. Aly Farag who has always pushed me to Think Big, and always continue to strive for the stars. Thank you all very much. 


\begin{abstract}
Early detection of human disease in today's society can have an enormous impact on the severity of the disease that is manifested. Disease such as Autism and Dyslexia, which have no current cure or proven mechanism as to how they develop, can often have an adverse physical and physiological impact on the lifestyle of a human being.

Although these disease are not fully curable, the severity handicaps that accompany them can be significantly reduced with the proper therapy, and thus the earlier that the disease is detected the faster therapy can be administered. The research in this thesis is an attempt at studying discriminatory shape measures of some brain structures that are known to carry changes from autistics to normal individuals. The focus will be on the corpus callosum.
\end{abstract}

There has been considerable research done on the brain scans (MRI, CT) of autistic individuals vs. control (normal) individuals to observe any noticeable discrepancies through statistical analysis. The most common and powerful tool to analyze structures of the brain, once a specific region has been segmented, is using Registration to match like structures and record their error. The ICP algorithm (Iterative Closest Point) is commonly used to accomplish this task. Many techniques such as level sets and statistical methods can be used for segmentation. The Corpus Callosum (CC) and the cortical surface of the brain are currently where most Autism analysis is performed. It has been observed that the gyrification of the cortical surface is different in the two groups, and size as well as shape of the CC.

An analysis approach for autism MRI is quite extensive and involves many steps. This thesis is limited to examination of shape measures of the $\mathrm{CC}$ that lend discrimination 
ability to distinguish between normal and autistic individuals from T1-weigheted MRI scans. We will examine two approaches for shape analysis, based on the traditional Fourier Descriptors (FD) method and shape registration (SR) using the procrustes technique.

MRI scans of 22 autistic and 16 normal individuals are used to test the approaches developed in this thesis. We show that both FD and SR may be used to extract features to discriminate between the two populations with accuracy levels over $80 \%$ up to $100 \%$ depending on the technique. 


\section{TABLE OF CONTENTS}

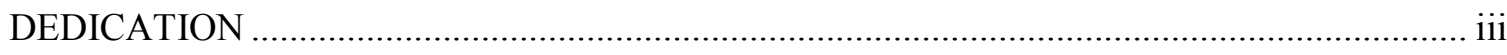

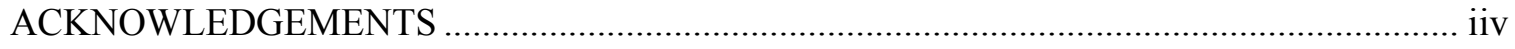

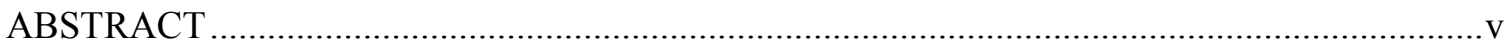

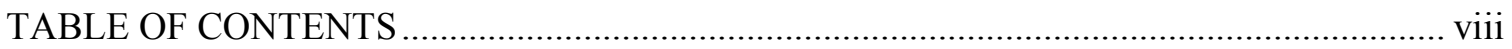

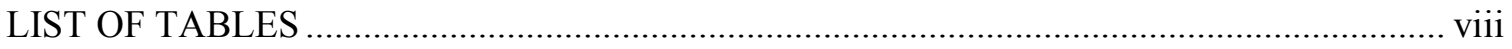

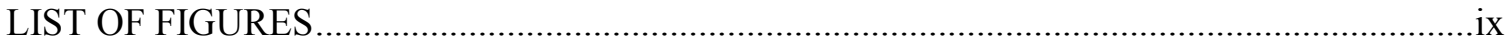

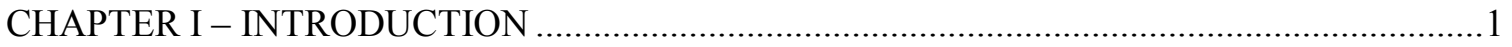

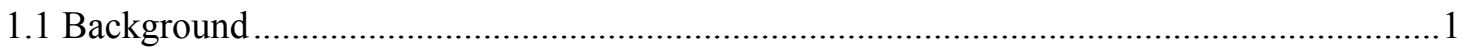

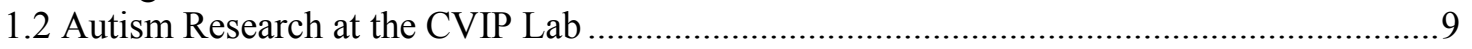

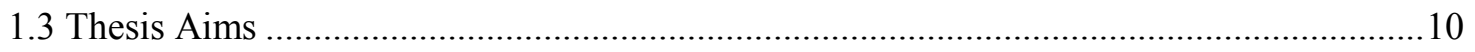

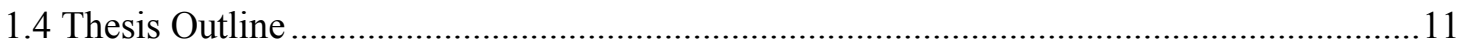

CHAPTER II - OVERVIEW OF IMAGE ANALYSIS FOR AUTISM ................................. 12

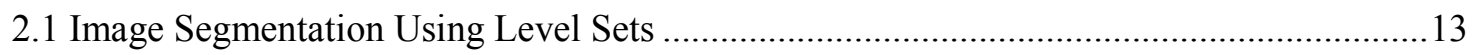

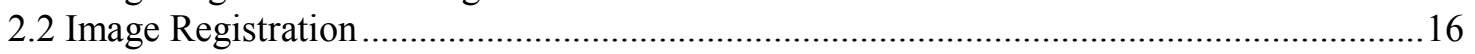

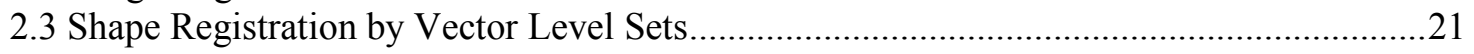

2.4 Shape Registration by the Procrustes Approach ..............................................................24

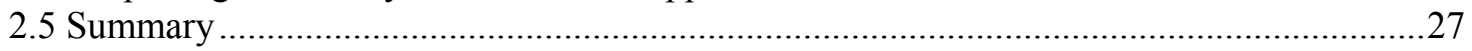

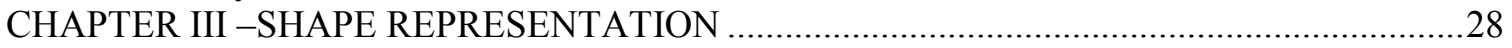

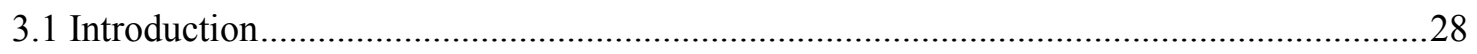

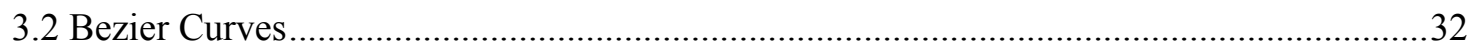

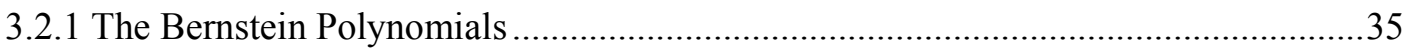

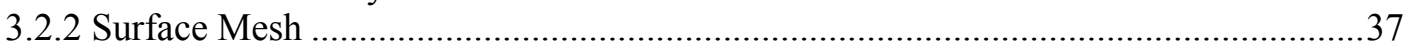

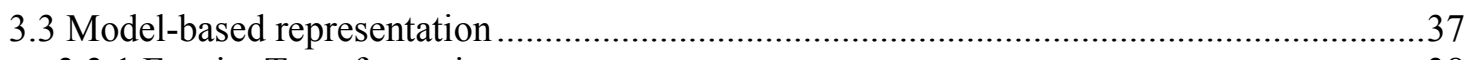

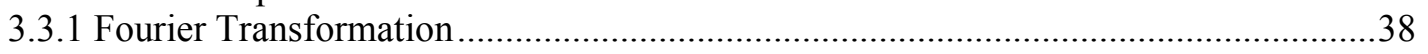

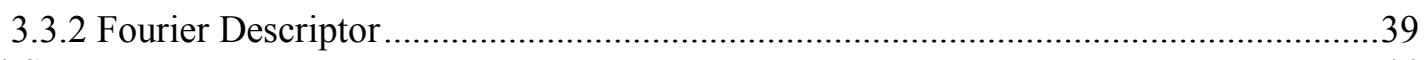

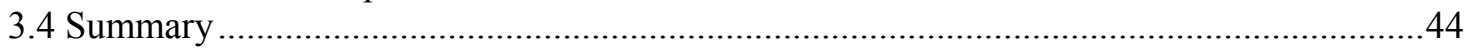

CHAPTER IV - SHAPE ANALYSIS OF THE CORPUS CALLOSUM ….............................45

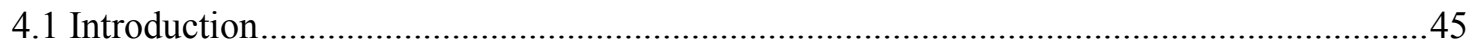

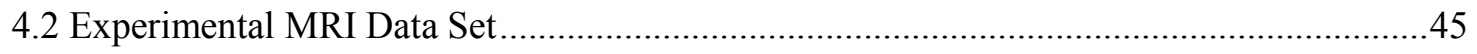

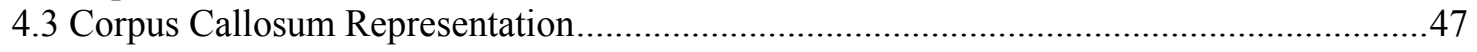

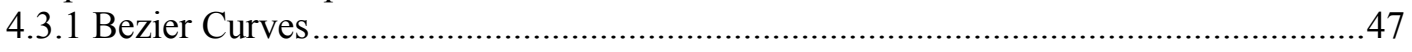

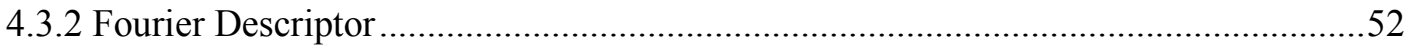

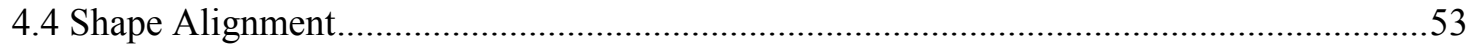

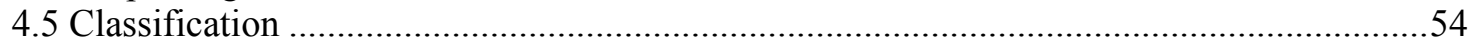

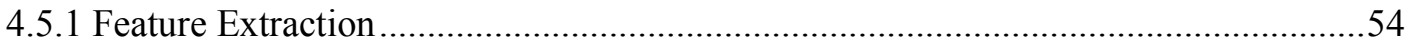

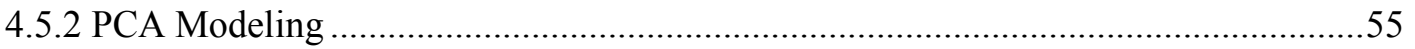

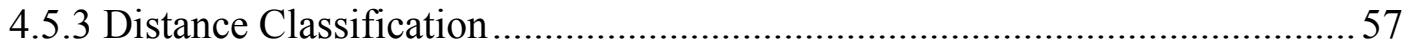

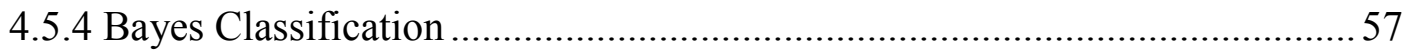

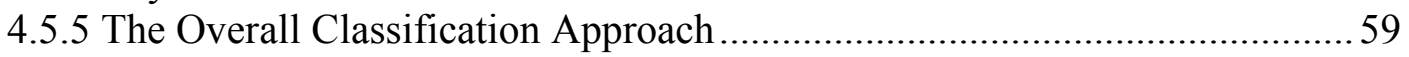

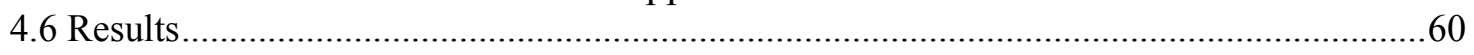

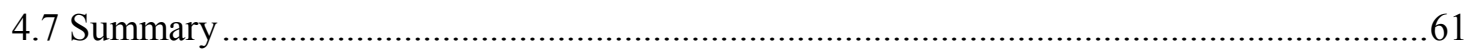

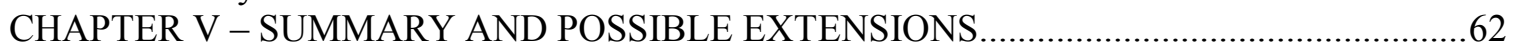

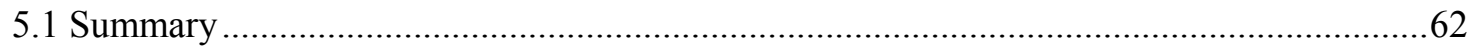

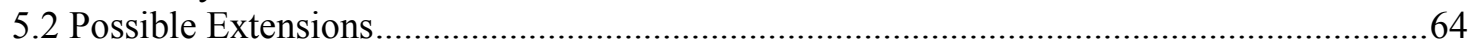

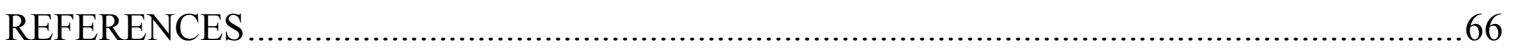

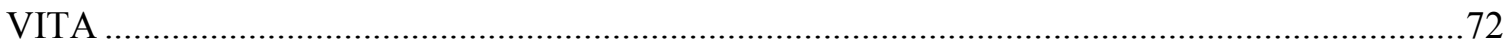




\section{LIST OF TABLES}

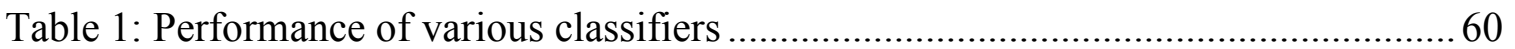




\section{LIST OF FIGURES}

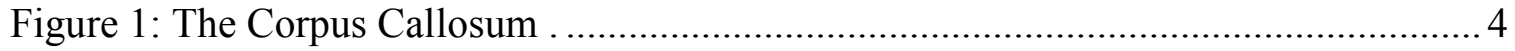

Figure 2: Corpus Callosum as shown in Sagittal plane.................................................. 5

Figure 3: Illustration of the brain limbic system ................................................... 8

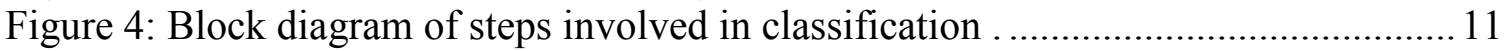

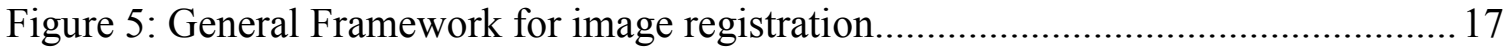

Figure 6: Block Diagram of major steps in image segmentation.................................. 20

Figure 7: Illustration of correspondences between model and target.............................. 20

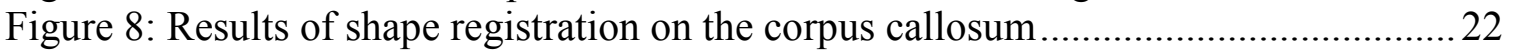

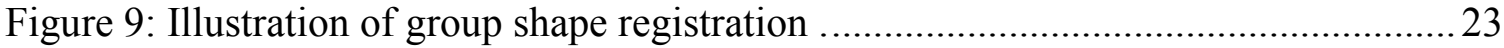

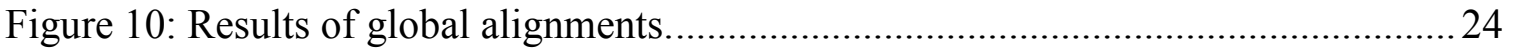

Figure 11: Procrastus distance tbween two shapes a and $b$.......................................... 25

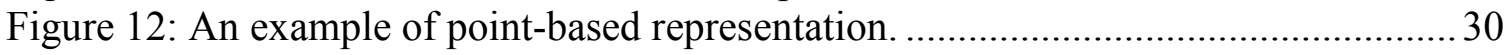

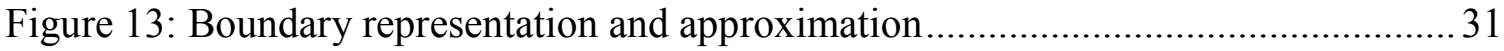

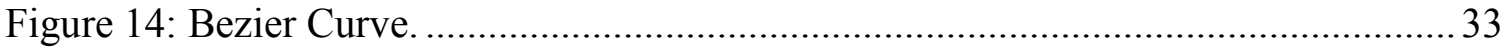

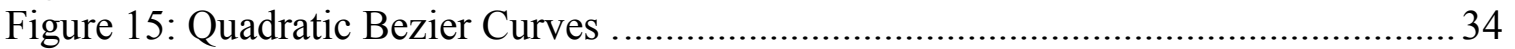

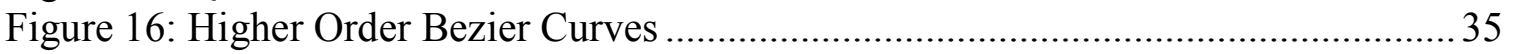

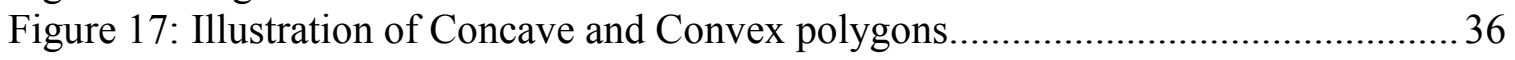

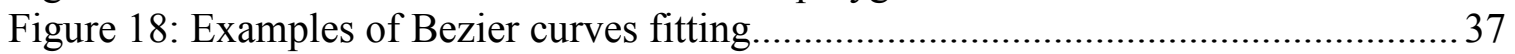

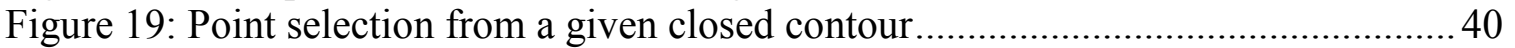

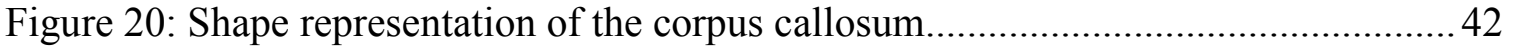

Figure 21: Interpolation of the corpus callosum shape. . ............................................. 43

Figure 22:Other interpolatoin results of more difficult cases. ..................................... 43

Figure 23: Fitting a Bezier curve to corpus callosum.............................48

Figure 24: Bézier curve of corpus callosum for autistic and normal subjects. ............... 49

Figure 25: Scatter plot of the Bezier coefficients of the corpus callosum .......................52

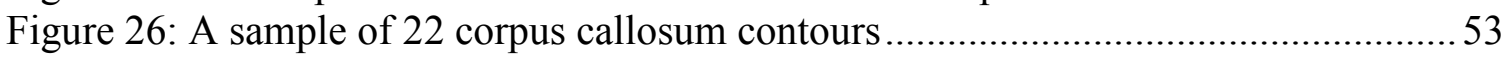

Figure 27: Individual and aligned Bezier Contours for 22 autistic subjects and their

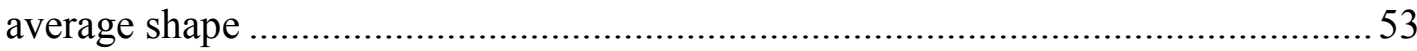

Figure 28: A sample of 16 corpus callosum contours of normal subjects and their

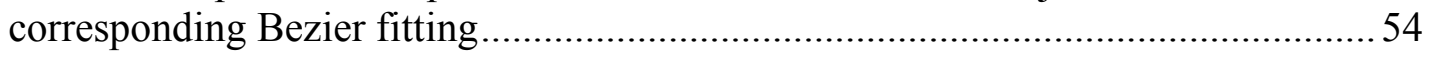

Figure 29: Individual and aligned Bezier contours for 16 normal subjects and their

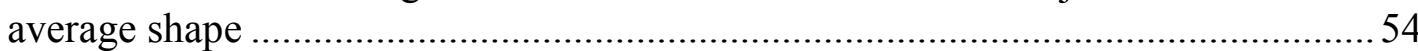

Figure 30: Given a set of training autistic corpus callosum rigidly registered. ................56

Figure 31: Given a set of training normal corpus callosum rigidly registered. ...............57

Figure 32:Other interpolatoin results of more difficult cases. ..................................... 58

Figure 33: Automatic classification of normal and autistic subjects from shape analysis of

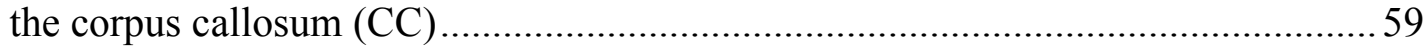




\section{CHAPTER 1}

\section{INTRODUCTION}

\subsection{Background}

Although it was once considered a rare disease, Autism has been shown to affect almost 1 in every 150 children [1][2][3]. Autism is a neurological disorder that is attributed with the impairment of a human beings social interaction, communication, and "typical" behavior. It generally is fully manifested during the first 3 years of life and adversely affects the function of the human brain. No study has effectively described the mechanism of this developmental disorder, and the exact parts of the brain involved are not fully understood either [2]. The parts of the brain necessary to understand autism are the Cortical Mini-columns, Corpus Callosum (CC), and the White Matter (WM).

Mini-columns are vertical iso/neo-cortical organization that link afferent, efferent and interneuronal signaling [33]. It has been theorized that supermumerary minicolumns expand the surface of the brain during encephalization (corticalization) while the cortical width is relatively unchanged, and that a substantial amount of added brain volume is given to minicoumn connectivity during this process with a change in the white/gray matter ratio [33]. Many neuroimaging studies have lead to the conclusion that autistic brains have a deficiency in the size of the $\mathrm{CC}$ and its corresponding regions along with an abnormal ratio of white/gray matter. 
Autism is a very complex developmental disease that impacts many functions of the brain and impairs development on many levels. The communication of an autistic individual (both children and adults) suffers greatly and affects almost every aspect of life from learning abilities to physical activities such as leisure sports. The disease does not have a specific ranking system for severity but the most severe conditions are complemented with cognitive deficits such as mental retardation, aggressive behavior, and self-injury. These impairments can last over the course of the lifetime of the individual and has a great impact on the people and environment that the individual interacts with [33]. The best treatment available for autistic individuals are educational, behavioral, and skill-oriented therapies that can possibly reduce the current condition, and are most effective when started at an early age.

Recently the neuropathology of autism has received great attention by the scientific community. Typically patients with autism have significant differences in several regions of the brain when compared to developing brains without autism. Head size was noticed as a correlated factor in children with autism, in a study by Kanner [7] over 60 years ago. Further studies have confirmed increased head circumference and brain size in autistic children. In other studies macrocephaly (head circumference larger than the 97th percentile of the population) was found in $20 \%$ of the autistic patients $[8][10][11][12][13]$. Modern neuroimaging has confirmed increased brain size in young autistic children [14][12]. However at the adolescent and adult level there were no significant differences in brain volume reported leading researchers to focus on the brain during development. [15][12]). Furthermore, the following specific parts of the brain 
have been analyzed under great scrutiny to find anomalies with nonautistic brains: the cerebellum, the brain stem, the corpus callosum, and the limbic system.

\section{The Cerebellum}

The cerebellum is located on the lower rear part of the brain. It controls the integration of different sensory perceptions and motor functions through neural connectors with the motor cortex.

In a study by Courchesne et al. [27] examining MRIs of patients with autism showed a reduction in the neocerebellar vermis, which would be consistent with the studies reporting reduced Perkinje cell size in autistic patients. MRI studies, as previously mentioned have shown an increased volume in brain size. Particularly notable was cerebellar white matter of children with autism, [15]. But, these reports have also found that the volume of cerebellar gray matter in autistic children did not differ from control subjects. Surprisingly, this difference was noticed in older patients. Allen and Courchesne have found (using functional MRI studies examining the cerebellum) abnormalities in cerebellar development of autistic patients which may create different problems for motor and attentional functioning [28].

A decreased amount of Perkinje cells in the cerebellar hemispheres, vermis, and cerebellum were found in almost all neuropathological studies of people with autism. This was first reported in Williams et al. [23] in the case of four individuals. Recently Ritvo et al. [24] found a decrease in the number of Perkinje cells in the vermis and cerebellar hemisphere in a study with three autistic males. Further studies by Lee et al. 
and Bailey et al. have confirmed and provided these same results [25][26]. On the other hand reports by Guerin et al. have shown no differences in the cerebellum of autistic patients [16]. However, they did find that the corpus collasum tends to be thinner.

\section{The Corpus Callosum}

The corpus callosum is the part of the brain which in mammals connects the left and the right hemisphere. It is the largest white matter structure in the brain containing over 200 million contralateral axona and as previously mentioned has been indentified in studies relating to autism.

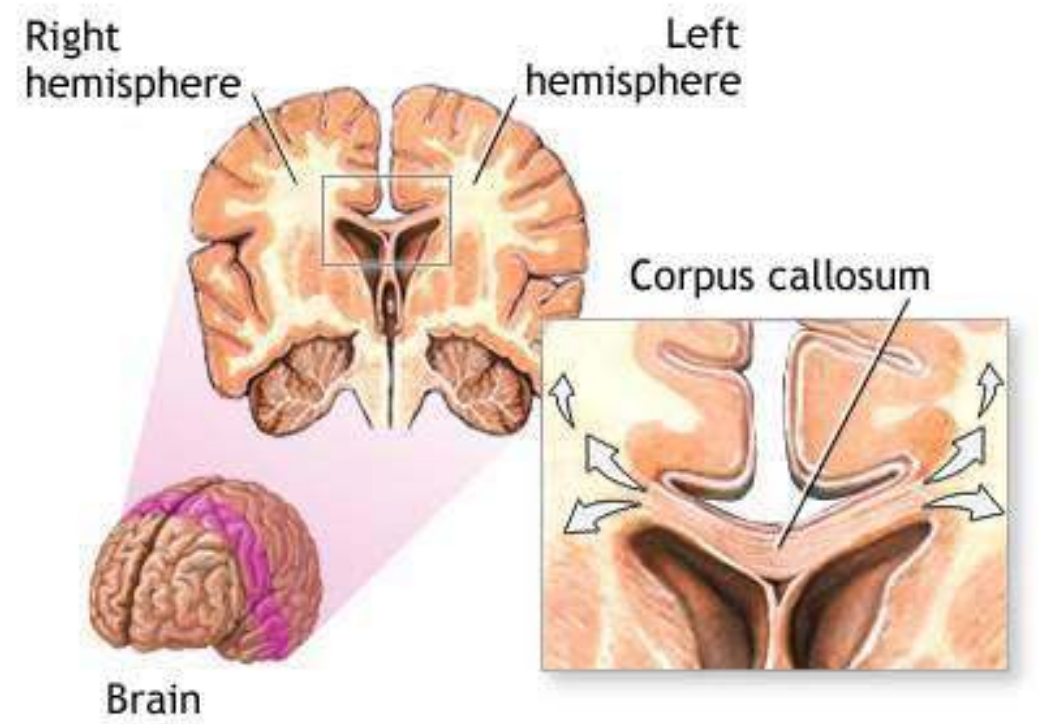

\section{FADAM.}

Figure 1: The Corpus Callosum. Adopted from [36].

According to Farag et al. geometric analysis of postmortem normal and autistic human subjects reveal distinctions in deformations in the corpus callosum (CC) that may be used for image analysis-based studies of autism. Preliminary studies showed that the 
$\mathrm{CC}$ of autistic patients is quite distinct from normal controls [33]. In a study of the microstructure of corpus callosum and cortical connectivity differences by Xiaoyan et al. has been found between two subjects. These abnormalities revealed "aberrant cortical connections between brain regions, which are consistent with the hypothesis of abnormal cortical connectivity in autism [35].

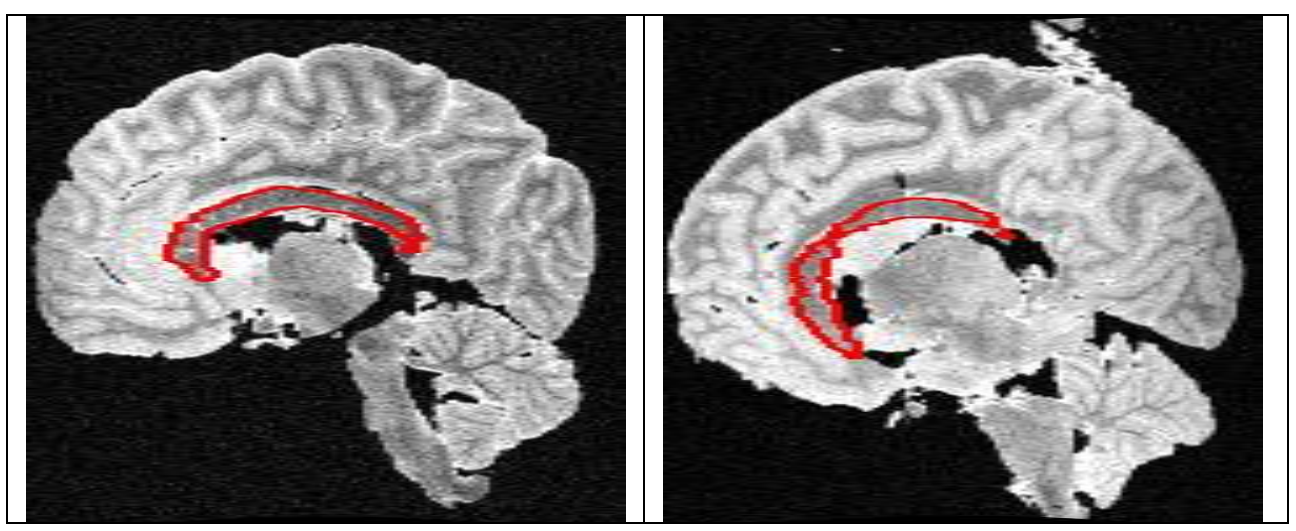

Figure 2: The Corpus Callosum as shown in saggital plane cross section of postmortem T1 weighted MRI scans of normal (left) and autistic individuals (right) [30].

\section{$\underline{\text { Brainstem }}$}

As with the cerebellum and Perkinje cells there are inconsistencies between pathological findings related to the brainstem in autism. According to a study by Bauman et al. [8], neurons in the inferior olive were much larger than normal in younger autistic patients, those between 9 and 12 years of age. But in older cases, 22 years of age and older, the neurons were found to be smaller and pale. In a more recent study by Bailey et al. they reported also having olivary abnormalities in five autistic patients. The lack of consistency between different findings of the pathological neuropathy could be due to 
these relatively small sample sizes. But in these studies they did not account for significant cofactors such as age, sex, socio-economic status, mental background, which could play a factor in these inconsistencies.

Although no direct correlation exists between the reported structural MRI findings and the neuropathological findings in autism, there are consistently replicable results which show an elevated brain volume in autism, particularly in younger patients. However, the other regions of the brain were identified, although with less certainty as being directly related to the abnormal neurological development associated with autism.

Therefore ultimately what is needed is more studies between neuroimaging and the neuropathological research in autism, specifically in the differences in how the brain grows and changes from youth to adulthood in patients with autism. Longitudinal studies will be necessary to show the path of brain development in people with autism. It is worth noting that autism research may benefit from new imaging modalities such as diffusion tensor imaging (DT-MRI). Specifically, this imaging modality offers a better ability to learn about the structure of white matter in patients with autism. In fact a study by Barnea-Goraly et al. has used DT-MRI to research white matter differences between several developmental disorders including.

An increased in brain size and gyrification are both present in encephalization. But, major changes in various key parts of brain structure show that a human being's brain is not just a larger version of an ape's. Usually increases in brain size related to the neocortex, come at the cost of other components. In a study by Ringo (1991) [32] the impossibility of maintaining the same "percent connectedness" with increasing numbers 
of gray matter neurons was noted. This is likely the result of losing longer corticocortical connectionse like the commissural pathways. So during the process of encephalization, cerebral white matter will increases at a faster rate than gray matter. Meanwhile cross sectional measurements of the corpus callosum area fall behind those for overall brain size. This is consistent with the thinner corpus callosum noted by Guerin et al. Since the corpus callosum forms the connection between the two hemispheres the disconnection between the hemispheres due to autism may be responsible for the emergence of cerebral dominance. This is supported by neuroimaging studies reporting a negative correlation between language lateralization and callosal size (Hines et al., 1992) [31]. It is noted that when processing of information takes place predominantly in one hemisphere, there is less need for interhemispheric information exchange.

Further research has shown that in patients with autism, there is an age dependent increase in brain size. The maximum growth seems to happen during the first year of life. Studies of brain size in adolescents and adult autistic patients are normal. Therefore autistic people seem to have an excessive brain growth early in life which can be followed by the loss of less efficient connections (long association fibers) that are constrained by the reduced aperture of the gyral window.

Subregions of the corpus collasum experiences area changes directly corresponding to sites of cortical development during the prenatal period, a process that is associated with myelination and fiber diameter expansion instead of the multiplying of fibers. During this time period, postnatal retractive events that cause abnormalities in the maturation of the $\mathrm{CC}$ and other cortical sturctures may cause the manifestation of autism and the 
concurrent problems associated with it. Analysis of early CC lesions and fiber reorganization, observed before maturation, may lead to novel acquistition techniques.

\section{$\underline{\text { Limbic system }}$}

The limbic system includes many of the cortical and sub-cortical brain structures. This includes the structures that are involved in emotion and memory association with emotions. These structures include: the amygdale, the hypothalamus, the orbito-frontal cortex, the sub-column, etc. Bauman and Kemper [9] noted a significant increase in cell packing and reduced cell size in the hypocampus, amygdale, subiculum, and other structures of the limbic system. The study was the result of a comparative analysis between six autistic cases (9-29 of age) and six age- and sex-matched controls.

Figure 3:

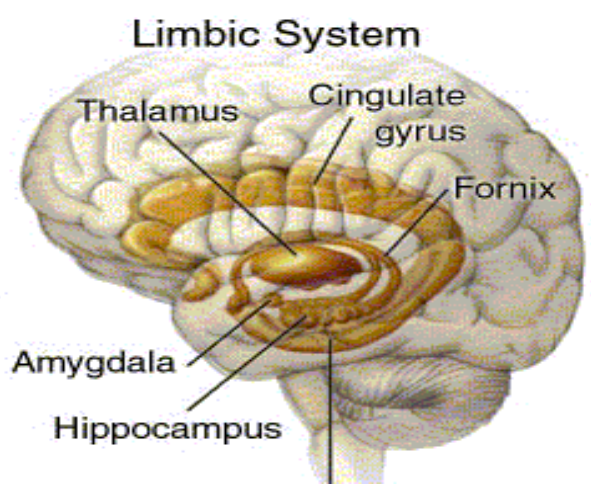

Illustration of the brain limbic system.

As previously mentioned MRI based studies have reported that the corpus callosum is smaller and thinner in autistic subjects [33]. However, it is not clear as of which part of the corpus callosum is abnormal. Most of the studies report a reduced posterior corpus callosum. However other studies including the one by Guerin et al. have found that the 
reduction was only of the anterior segment [33]. A recent MRI study by Vidal et al. [21] of 24 boys with autism has shown aberrant connections between cortical regions when compared to the controls. The study has also reported reductions in the total colossal area and the anterior third of the corpus callosum in autistic patients.

Unfortunately MRI studies of the hippocampus and the amygdale have reported inconsistencies in their findings as well. The volume of the limbic structures either increased, decreased, or in autistic patients were similar relative to control cases [22].

\subsection{Autism Research at the CVIP Lab}

The Computer Vision and Image Processing Laboratory (CVIP Lab) has been involved in image analysis reach of autism since 2004 in collaboration with Dr. Manuel Casanova, Department of Psychiatry, University of Lousiville. The underlying hypothesis of these activities is two fold: a) pathological minicolumns disturbances at the micro level are manifested in macroscopic variations in the $\mathrm{WM}, \mathrm{CC}$ and other brain structures which can be observed in high resolution T1-weighted MRI scans; and b) variational image modeling and scale space techniques produce robust characterization of the gyrification window and the topology of the corpus callosum and possibly the ventricles and the hippocampus, leading to discriminatory measures of autistic and normal brains.

A number of techniques for image segmentation and classification have been developed (e.g., [33][34]). These approaches were implemented on a postmortum MRI study (T1-weighted) of 22 autistic and 16 normal brains. 
This thesis is a step along the same directions which focuses on shape analysis of the CC.

\subsection{Thesis Aims}

The main goal of this thesis is to develop robust discriminatory measures that can distinguish the brains of autistic and normal subjects from T1-weighted brain imaging. Specifically, the domain of the Autism research at the CVIP Lab includes: 1) creating a standard image representation of T1-weighted MRI scans for the population involved in this study; 2) developing a level set approach for simultaneous segmentation and registration of the full brain and possibly the cortical surface, corpus callosum, ventricles and the hippocampus; and 3) using pattern analysis methodologies, in particular the scale space approach, to extract the most salient and discriminatory features between the brains of autistic and normal subjects.

Within this framework, a number of tools have been developed over the years which serve as kernel or stepping stone for further developments. Therfore, this thesis will build upon the tools at the CVIP Lab and will have the following focus: 1) study shape models that are suitable for the corpus callsoum; 2) study the features or measures from these shape models that lend benfit for discrimination between populations; and 3) test the effectivness of these shape measures for discriminating normal and autistic populations from a clinical MRI study. Figure 4 illustrates the flow of steps used in the thesis research. 
The contribution of this thesis is two fold: a) developing a systematic approach for shape modeling of the corpus callosum; and b) developing an approach for automatic classification of autistic vs. normal subjects from T1-weighted MRI data.

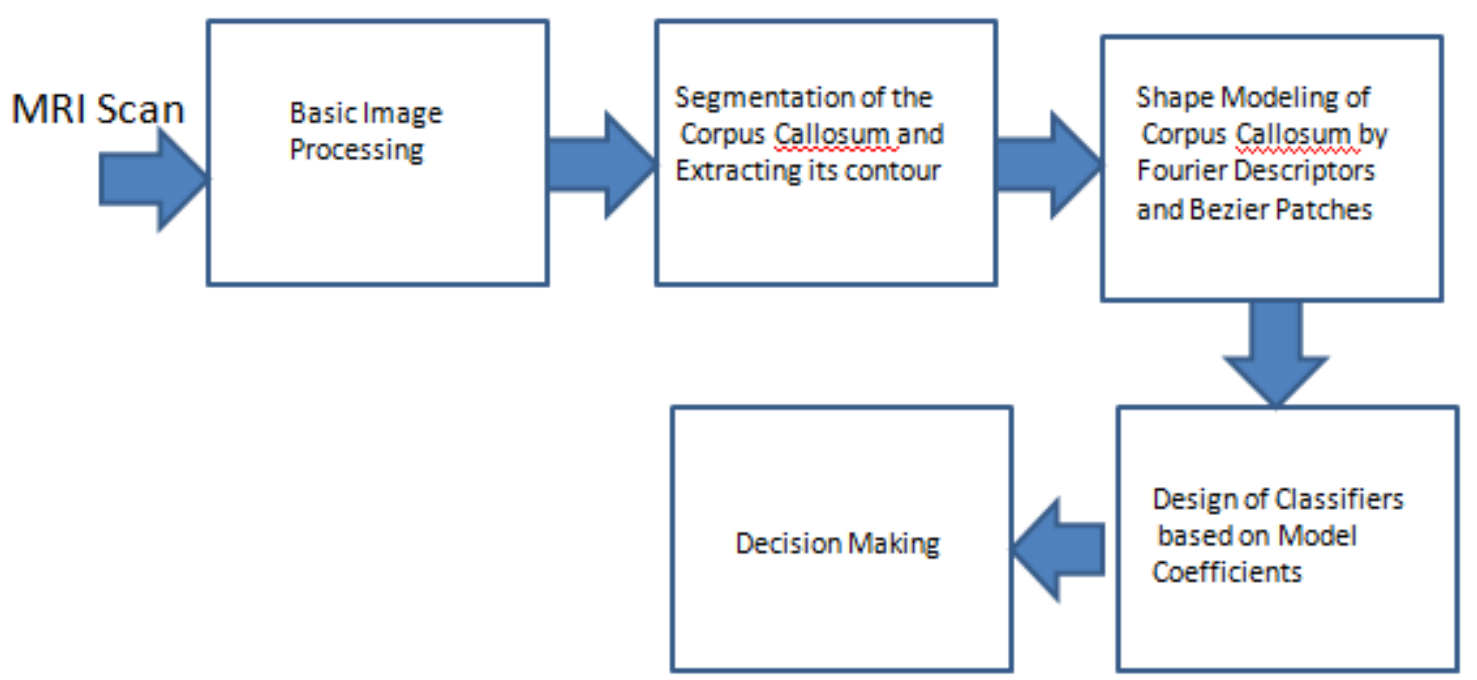

Figure 4: Block diagram of steps involved in classification of autistic vs. normal subjects from MRI data.

\subsection{Thesis Outline}

Chapter 2 examines some of the basic methods for brain imaging analysis. It includes a summary of the major techniques used in this thesis. Chapter 3 considers two approaches for shape respresentation of the $\mathrm{CC}$ based on the Fourier Descriptors and the Bezier Patches polynomial fitting. Chapter 4 examines the use of the shape representation methods in Chapter 3 for study of autism. Chapter 5 provides a summary and possible extensions of the research in this thesis. 


\section{CHAPTER 2}

\section{OVERVIEW OF IMAGE ANALYSIS FOR AUTISM}

This chapter describes typical image analysis steps in the study of autism. As the approaches vary based on the data at hand, we will not attempt a comprehensive survey. We will limit the study to structural imaging based on T1-weighetd MRI scans. As stated in the introduction, the cortical surface and corpus callosum have received particular focus in autism research based on neuroimaging. The basic steps on image analysis involve image segmentation, registration and classification (Figure 4). At the CVIP Lab, statistical and level sets approaches have been used extensively for segmentation of the cortical surface and for isolating the corpus callosum (e.g., [33][34]). Similarly, a number of approaches have been developed for shape registration which are of particular applicability to structures like the corpus callosum (e.g., [33][37][38][40]).

We will focus our survey here to methods based on level sets; the mathematical developments of these approaches are very involved and well described in the $\mathrm{PhD}$ dissertations of two CVIP Lab graduates, Dr. Hossam Abdemunim and Dr. Rachid Fahmi (e.g., [39][41]). The segmentation and the registration approaches of Abdelmunium and Farag [38] and Fahmi and Farag [40] have been used in this thesis. We will highlight some standard approaches for registration in the computer vision and biomedical imaging literature which is of particular interest to the research in this thesis; specifically, the Mutual Information approach for intensity registration, ICP algorithm for rigid registration and the Procrustus technique for shape registration. 


\subsection{Image Segmentation Using Level Sets}

Level set methods were first introduced by Osher and Sethian, 1988 [42] and has found wide spread applications in computer vision and biomedical image analysis. The basic idea is to represent contours as the zero level set of an implicit function defined in a higher dimension, referred to the level set function, and to evolve the level set function according to a partial differential equation (PDE). This method presents several advantages (e.g., Sethian, 1999 [43]) over the traditional parametric active contours. The contours represented by the level set function may break or merge naturally during the evolution, and changes are automatically handled. Another advantage is that the level set function always remains a function on a fixed grid, which allows efficient numerical schemes. The variational level set methods are more convenient than pure PDE driven level set methods because region-based information and shape-prior information are directly formulated in the level set domain. Hence, variational level set methods produce more robust results. For instance, Chan and Vese, 2001 [44] proposed an active contour model using a variational level set formulation.

Deformable models have had great success in medical imaging and computer vision. However, the disadvantage of this method is that the initial contour should be close to the final one. The method has some problems with topological changes of a complex structure (e.g., Abdelmunim and Farag, 2005 [45][46]). A signed distance map is used to handle complex rigid transformations with different scaling $\left(s_{x}, s_{y}, s_{z}\right)$, rotation $\left(\theta_{x}, \theta_{y}, \theta_{z}\right)$, and translation $\left(t_{x}, t_{y}, t_{z}\right)$ parameters of the shape registration.

The distance maps result in a more adequate energy function that obtains the transformation parameters. Also a shape-based PDE approach is included in this method. Hence, we do not need to tune the weighting coefficients. The first function is built as a function of the signed distance 
maps of the training shapes in a form of a parametric shape model. The second one is the segmentation of the region of interest based on the color value. The last function represents the evolving shape resulting from the combination of the color and the shape information. The energy function is obtained to measure the difference between the shape model and the color functions. The shape and the pose parameters are required to minimize this energy in a gradient descent approach (e.g., Farag and Abdelmunim 2004 [37]).

Farag and Abdelmunim [45][46] introduced a new level function defined as a vector distance rather than a scalar method. The level set function $\Phi$ is used to represent the evolving region. These representing shapes are invariant to translation and rotation. Given a curve/surface $V$ that represents boundaries of a certain shape, the following level set function can be defined as, $\Phi: R^{4} \rightarrow R^{3}$ where $\Phi(X, t)=\left[\phi_{1}(X, t), \phi_{2}(X, t), \phi_{3}(X, t)\right]^{T}$. It is defined as the minimum Euclidean distance between the point $X=[x, y, z]^{T}$ and a curve/surface $V$. The evolving region is a propagating front embedded as the zero level or a higher dimensional function $\Phi$ [45]. The continuous change of the projections of $\Phi$ is described as

$$
\frac{d}{d t} \phi_{i}+\left|\nabla \phi_{i}\right| F_{i}=0 \quad i=1,2,3
$$

where $F$ is a vector velocity function depending on the local curvature of the front and on the external features related to the input image [45]. The parameter $\Phi$ deforms iteratively according to $F$. The position of the front is given at each iteration step by using the following equation:

$$
\Phi(x, y, z, t)=0
$$

F can be defined as

$$
F=\left[v-\varepsilon k_{1}, v-\varepsilon k_{2}, v-\varepsilon k_{3}\right]^{T}
$$


where $v=1$ or $v=-1$ for contracting or expanding the front, respectively, $\varepsilon$ is a smoothing coefficient smaller or equal to 1 , and $k_{i}$ is the local curvature defined for the corresponding projection function $\Phi_{i}$ where $i=1,2,3$.

The intensity segmentation is described by the function $\Phi_{i}$ which changes based on (Eq. 2.1). If the point belongs to the associated object, the front region expands, otherwise it contracts. The point classification is based on the Bayesian decision at point at $X$. The parameter $v_{i}$ for each point is replaced by the function $v_{i}(X)$ can be defined as follow:

$$
v_{i}(X)=\left\{\begin{array}{lll}
-1 & \text { if } & \pi_{o} p_{o} I(X) \geq \pi_{b} p_{b} I(X) \\
+1 & \text { if } \text { otherwise }
\end{array}\right.
$$

where $\pi$ is the region prior probability, $p($.$) is the probability density function (pdf) for the object$ $(o)$ and the background $(b)$, and $I$ is the image data. Each region is defined by a Gaussian distribution with adaptive parameters as follows [45]:

$$
\begin{gathered}
\pi_{o}=\frac{\int_{\Omega} H\left(-\phi_{g}\right) d \Omega}{\int_{\Omega} d \Omega}, \pi_{b}=\frac{\int_{\Omega} H\left(\phi_{g}\right) d \Omega}{\int_{\Omega} d \Omega} \\
\mu_{o}=\frac{\int_{\Omega} H(-\phi) I d \Omega}{\int_{\Omega} H(-\phi) d \Omega}, \mu_{b}=\frac{\int_{\Omega} H(\phi) I d \Omega}{\int_{\Omega} H(\phi) d \Omega} \\
\sigma_{o}{ }^{2}=\frac{\int_{\Omega} H\left(-\phi_{g}\right)\left(I-\mu_{o}\right)^{2} d \Omega}{\int H\left(-\phi_{g}\right) d \Omega}, \sigma_{b}{ }^{2}=\frac{\int_{\Omega} H\left(\phi_{g}\right)\left(I-\mu_{b}\right)^{2} d \Omega}{\int H\left(\phi_{g}\right) d \Omega}
\end{gathered}
$$


where $H$ is the Heaviside step function.

$\Phi_{1}, \Phi_{2}$, and $\Phi_{3}$ are the projections of the distance in the coordinates directions negative inside the curve/surface, positive outside and zero on the boundary ([45][46]). The algorithm can be summarized as follows:

\section{Level Set Image Segmentation Algorithm: Farag-Abdelmunim [46]}

Step 1: Manually select the initial seeds inside the region of interest.

Step 2: For the bi-model in this work, assume object and background classes have Gaussian distribution.

Step 3: Obtaining edges.

Step 4: Iteratively estimate the mean and standard deviation of the object and background (Eqs. 2.5- 2.7).

Step 5: For each pixel do the Bayesian decision (Eq. 2.4)

Step 6: Repeat steps 4 and 5 until the iteration is ended.

\subsection{Image Registration}

Image registration is a fundamental step in image analysis; in fact, it the logical step behind essentially all pattern recognition and machine learning techniques which relates a measurement to a typical reference. From the mathematical point of view, 
registration aligns a target $(\mathrm{T})$ to a model $(\mathrm{S})$ such that a certain similarity criterion is optimized. Figure 3 illustrates the basic steps in image registration.

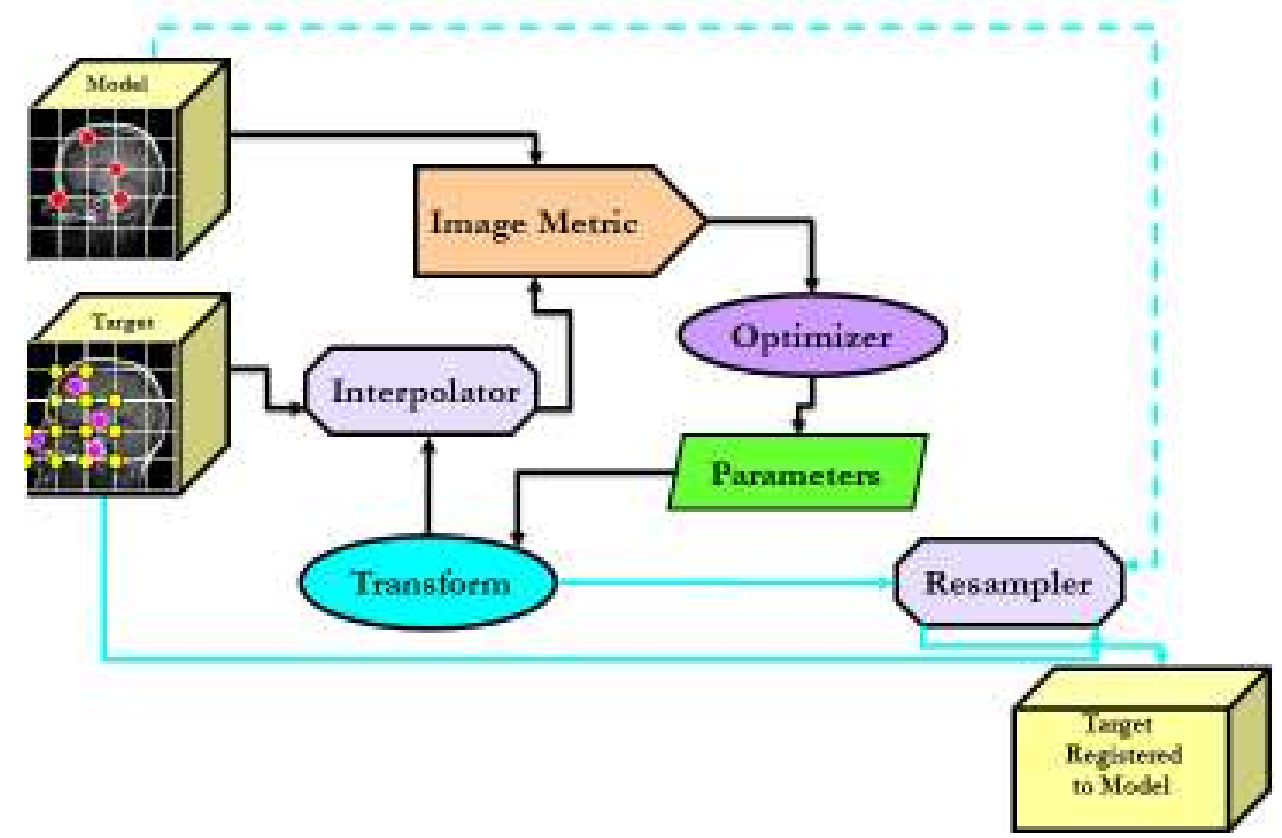

Figure 5: General framework for image registration. The Target image $T$ is registered to the model image $M$ in accordance to a certain similarity measure.

Among the similarity metrics are the mean square error, the normalized cross correlation and the mutual information [47]. The transformation $\mathbf{T}(\mathbf{p} ; \boldsymbol{\Theta})$ that takes the target image to the model involves three main components: translation, rotation and scaling. The transform itself may be rigid for non deformable objects or elastic which allows a degree of variability in the either the target or the model or both. Optimization approaches include brute force search, gradient descent, Levenberg-Marquardt among various other numerical approaches. Interpolators include Nearest Neighbor, Bilinear and B-Spline [47]. Let model (reference or fixed) image or volume be $\mathbf{F}(\mathbf{x})$ and the target (moving) image or volume be $\mathbf{M}(\mathbf{x})$. Let $\mathbf{x}$ be coordinates of the volume, $\mathbf{T}$ be 
transformation from coordinate frame of the fixed volume to moving volume, and $\mathbf{M}(\mathbf{T}(\mathbf{x})$ ) be target pixel (voxel) associated with model pixel (voxel) $\mathbf{F}(\mathbf{x})$. We can define the following similarity measures:

\section{Mean square error (MSE):}

$$
S(\Theta \mid F, M, \mathbf{T})=\frac{1}{N} \sum_{i=1}^{N}\left[F\left(x_{i}\right)-M(\underbrace{\mathbf{T}\left(p_{i}, \Theta\right)}_{q_{i}})\right]^{2}
$$

In carrying out the MSE measure, the mean squared difference over all the pixels in an image is calculated, intensities are interpolated for the target image, and for gradientbased optimization, derivative of metric is also required. This measure is applicable for images of same modality (i.e., MRI over MRI, CT over CT, etc.).

\section{The Normalized Correlation:}

$$
S(\Theta \mid F, M, \mathbf{T})=\frac{\sum_{i=1}^{N} F\left(p_{i}\right) \cdot M\left(T\left(p_{i} ; \Theta\right)\right)}{\sqrt{\sum_{i=1}^{N} F^{2}\left(p_{i}\right) \cdot \sum_{i=1}^{N} M^{2}\left(T\left(p_{i} ; \Theta\right)\right)}}
$$

This measure is also applicable for registering images/volumes of similar modalities.

\section{The Mutual Information:}

$$
\begin{aligned}
& \hat{T}=\arg \max _{T} I(F(x), M(T(x))) \\
& I(F(x), M(T(x))) \equiv h(F(x))+h(M(T(x)))-h(F(x), M(T(x)))
\end{aligned}
$$

Where I(.) is the information and $\mathrm{h}($.$) is entropy. The term h(M(T(x)))$ encourages transformations that project the model $(\mathrm{M})$ into complex parts of the target $(\mathrm{T})$. The last term of MI equation contributes when the model and target are functionally related [48]. 
Together, the last two terms of MI equation identify transforms that find complexity and explain it well. To find maximum of mutual information, we calculate its derivative:

$$
\frac{d}{d T} I(T) \approx \frac{d}{d T} h *(F(x))+\frac{d}{d T} h *(M(T(x)))-\frac{d}{d T} h *(F(x), M(T(x)))
$$

The derivative of fixed/model volume $F(x)$ is 0 , because it is not a function of $\mathbf{T}$. Entropies depend on the covariance of probability density functions, which may be estimated numerically using different methods (e.g., the linear model or the Parzen window [49]). The overall process of mutual information maximization is illustrated in Figure 6.

The MI-based approach works on multimodal imaging; hence, we may register a CT scan to an MRI or an ultrasound scan to a CT or MRI. This measure is also more accurate than either the MSE or the normalized correlation approaches. However, it is more extensive in terms of computation than other approaches. We will examine below a rigid registration approach that is based on distance transformation and we will focus on a well-established algorithm for optimization of the distance between the target and model images known as the iterative closest point (ICP) algorithm [50].

\section{The ICP Algorithm [50]:}

Given a model shape, the maybe represented as: Point Sets, Line Segment Sets, Implicit Curves, Parametric Curves, Triangle Sets, Implicit Surfaces, or Parametric Surfaces. Given a target or scene shape which is represented as a point set, the target shape may correspond to the model shape. It is required to estimate the optimal rotation, translation and scaling that aligns or registers the target shape to the model shape. 


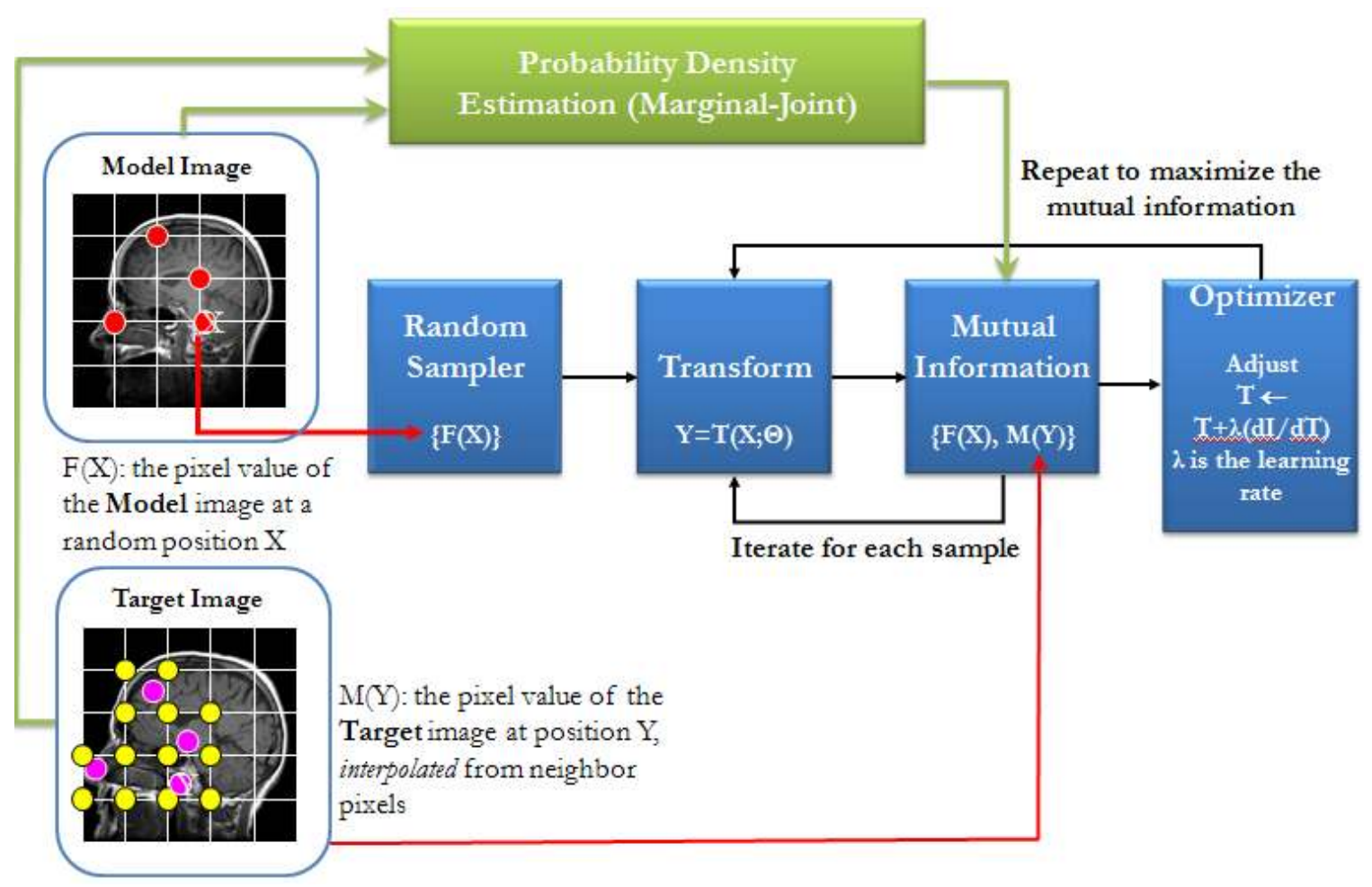

Figure 6: Block diagram of the major steps in image segmentation using maximization of the mutual information approach.

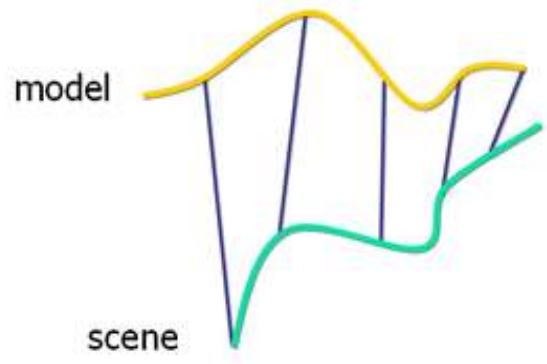

Figure 7: Illustration of correspondences between model and target (scene) shapes in the process of rigid registration.

The ICP algorithm registers the scene to the model shapes by minimizing, iteratively, the Euclidian distance between them. The basic steps in obtaining the translation, rotation and scaling using the ICIP algorithm are as follows: 


\section{Basic Steps in the ICP Algorithm}

1. Begin with initial rotation, translation and scaling (initial value for registration parameters).

2. Fix the model shape and start moving the scene shape by applying the initial registration parameters. i.e. scale, rotate and then translate.

3. Compute the error metric that reflects the dissimilarity of the scene shape from the model shape.

4. If the error is minimum, we have correctly aligned the scene shape to the model shape, return with the aligned scene shape.

5. Else, calculate the new values for the registration parameters and go back to step 2 with the new parameter values.

The ICP algorithm does not work properly on intensity representations and it is usually very slow especially with large number of points and in higher dimensions.

\subsection{Shape Registration by Vector Level Sets}

Abdelmunim and Farag (e.g., [45][46]) introduced a level set-based method for rigid and elastic shape registration. The approach will be briefly summarized here. Given two shapes represented by the vector functions $\Phi 1$ and $\Phi 2$. A transformation A with scales, rotation and translation is to be calculated to transform the first object to the second. The following dissimilarity measure the difference between the vector and the other scaled one:

$$
r=S R \Phi_{1}(X)-\Phi_{2}(A)
$$

The following energy is formulated as a sum of squared differences

$$
E=\int_{\Omega} \delta_{\epsilon} r^{T} r d \Omega
$$


Where the delta is an indicator function with value 1 inside the shape and zero otherwise. The two shapes are aligned by minimizing the energy function using various approaches including the gradient dissent method.
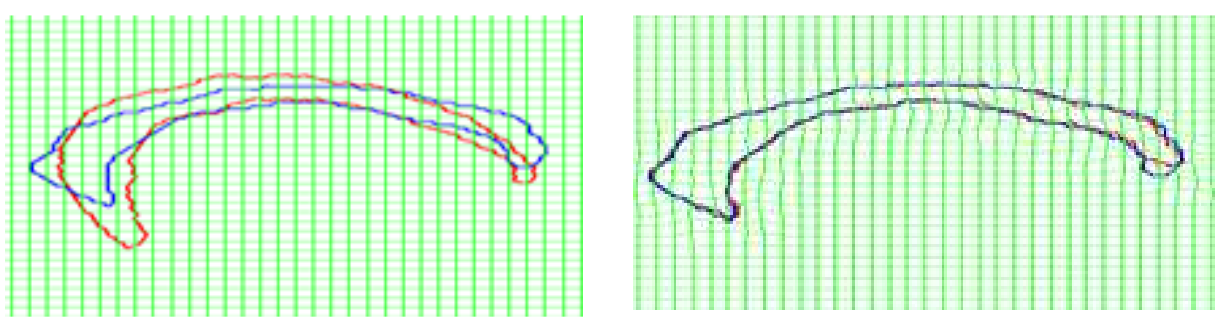

Figure 8: Results of shape registration on the corpus callosum using the Abdelmunim and Farag Algorithm [38]. Rigid registration/alignment on the left and elastic registration is on the right.

The approach may be used for aligning several shapes with respect to the mean shape as illustrated in Figure 9 (e.g., [46]). The training shapes (e.g., segmented corpus callosum) are jointly registered with an evolving mean shape to find the corresponding global transformations $\mathrm{A}_{1}, \ldots, \mathrm{A}_{\mathrm{n}} \cdot$ The dissimilarity measure is used as follows:

$$
r_{i}=S_{i} \Phi_{M}-\Phi_{i}\left(A_{i}\right)
$$

The energy function will be:

$$
E\left(\Phi_{M}, \Phi_{1}, \ldots, \Phi_{n}\right)=\sum_{i=1}^{n} \int_{\Omega} \delta_{\epsilon} r_{i}^{T} r_{i} d \Omega
$$




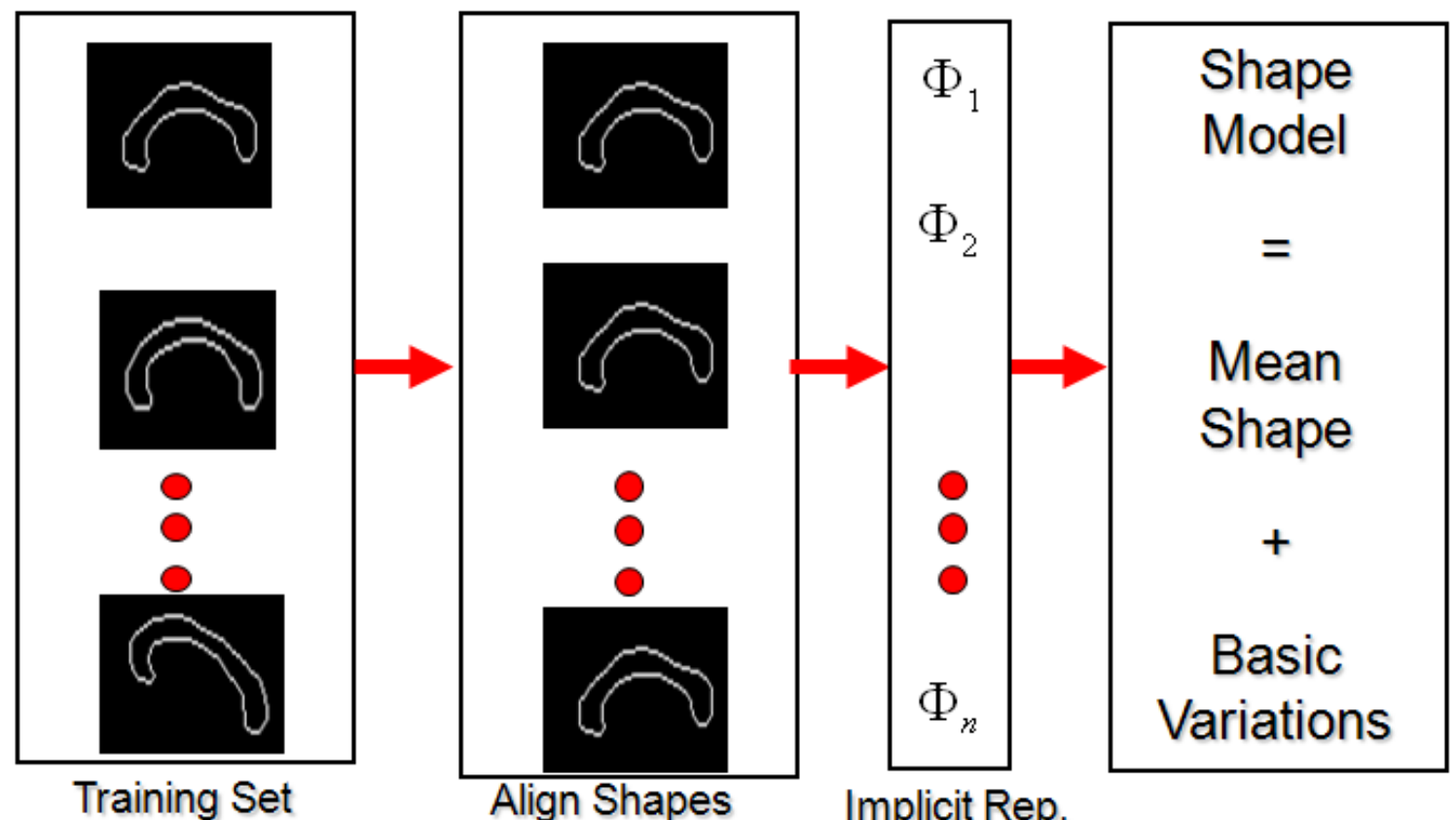

Figure 9: Illustration of group shape registration using vector level set function [38].

The transformation parameters are calculated using the gradient descent as follows (e.g., [39]):

$$
\begin{aligned}
& \frac{d}{d t} s_{i}=2 \int_{\Omega} \delta_{\epsilon} r_{i}^{T}\left[\nabla_{s_{i}} S_{i} \Phi_{M}-\nabla \Phi_{i}\left(A_{i}\right)^{T} \nabla_{s_{i}} A_{i}\right] d \Omega \\
& \frac{d}{d t} \theta_{i}=2 \int_{\Omega} \delta_{\epsilon} r_{i}^{T}\left[\nabla \Phi_{i}\left(A_{i}\right)^{T} \nabla_{\theta_{i}} A_{i}\right] d \Omega \\
& \frac{d}{d t} t_{i}=2 \int_{\Omega} \delta_{\epsilon} r_{i}^{T}\left[\nabla \Phi_{i}\left(A_{i}\right)^{T} \nabla_{t_{i}} A_{i}\right] d \Omega
\end{aligned}
$$

where

$$
\begin{aligned}
& s_{i} \in\left\{s_{x} . s_{y}, s_{z}\right\}_{i}, \\
& t_{i} \in\left\{t_{x} . t_{y}, t_{z}\right\}_{i}, \\
& \text { and } \theta_{i} \in\left\{\theta_{x} . \theta_{y}, \theta_{z}\right\}
\end{aligned}
$$

The average function will evolve according to the following equation: 


$$
\frac{\partial}{\partial t} \Phi_{M}=\sum_{i=1}^{n}\left(\nabla_{\Phi_{M}} \delta_{\epsilon} r_{i}^{T} r_{i}+2 \delta_{\epsilon} S_{i} r_{i}\right)
$$

The shape model is a function of the registered training shapes:

$$
\Phi_{p}=\Phi_{M}+\sum_{i=1}^{n} w_{i}\left(\Phi_{i}^{t}-\Phi_{M}\right)
$$

The shape parameters $\mathbf{w}$, need also to minimize the energy function to process the registration.

Various implementation details and the use of the shape models for registering shapes as well as shape-based segmentation are in [38][40]. Figure 10 shows the global registration of a number of corpus callosum shapes.
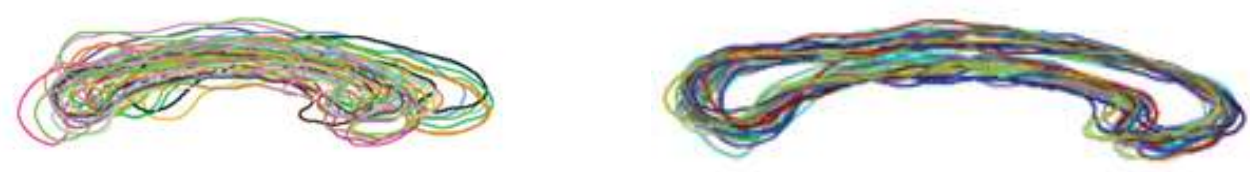

Figure 10: Results of global alignments of a number of corpus callosum using Shapebased approach (adopted from [38]).

Various approaches may be used for co-registering the corpus callosum Contours (e.g., [38-41][51]).

\subsection{Shape Registration by the Procrustes Approach}

Figure 11 illustrates the Procrustes distance between two shapes $a$ and $b$. There are several preprocessing steps to determine the Procrustes distance between two shapes (e.g., [51]):

1. Compute the centroid of each shape. 
2. Rescale each shape to have equal size.

3. Align with respect to position the two shapes at their centroids.

4. Align with respect to orientation by rotation.
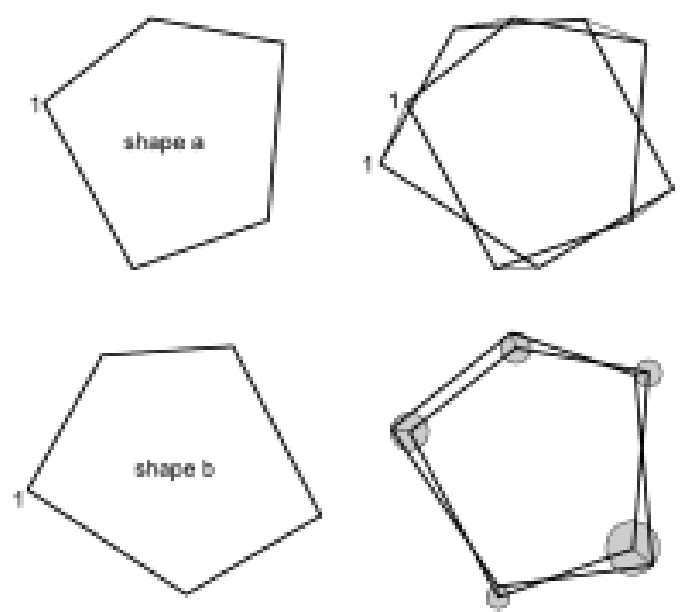

Figure 11: Procrustes distance between two shapes aandb.

The Procrustes distance $\left(M^{2}\right)$ between two shapes $x=\left(x_{x i}, x_{y i}\right)$ and $y=\left(y_{x i}, y_{y i}\right)$ is a least-squares type of metric of the form:

$$
M^{2}=\|x-y\|^{2}
$$

Procrustes distance-based rigid registration between two shapes usually involves minimizing the expression $\|T(x)-y\|^{2}$, where $T$ in the Euclidean case is

$$
T\left(\begin{array}{l}
x \\
y
\end{array}\right)=\left[\begin{array}{cc}
a & -b \\
b & a
\end{array}\right]\left[\begin{array}{l}
x \\
y
\end{array}\right]+\left[\begin{array}{l}
t_{x} \\
t_{y}
\end{array}\right]
$$


The term $\|T(x)-y\|^{2}$ can simply be differentiated with respect to the parameters $a, b, t_{x}$ and $t_{y}$, to determine the optimum parameters that minimize the expression $\|T(x)-y\|^{2}$. The actual closed-form solutions of the parameters are [52]:

$$
\begin{aligned}
& a=(x \cdot y) /|x|^{2}, \quad b=\left(\sum_{i} x_{x i} y_{y i}-x_{y i} y_{x i}\right) /|x|^{2} \\
& \left.t_{x}=(1 / n) \sum_{i} y_{x i}, \quad t_{y}=(1 / n) \sum_{i} y_{y i}\right)
\end{aligned}
$$

\section{Shape Warping}

Suppose we wish to warp a 2D shape $s=\left(x_{1}, y_{1}, \cdots, x_{n}, y_{n}\right)^{T}$, so that the set of $n$ control points $\boldsymbol{x}_{i}=\left[x_{i}, y_{i}\right]$ are mapped to new positions, $x_{i}^{\prime}=\left\{x_{i}^{\prime}, y_{i}^{\prime}\right\}$. We require a continuous vector-valued mapping function, $\boldsymbol{f}$, such that $\boldsymbol{f}\left(\boldsymbol{x}_{i}\right)-\boldsymbol{x}_{i}^{\prime}$. This function is a type of forward-mapping method. One such transformation is

$$
\left[\begin{array}{l}
x_{i}^{\prime} \\
y_{i}^{i}
\end{array}\right]=s\left[\begin{array}{ll}
a & b \\
c & d
\end{array}\right]\left[\begin{array}{l}
x_{i} \\
y_{i}
\end{array}\right]+\left[\begin{array}{l}
t_{x} \\
t_{y}
\end{array}\right]
$$

The Matlab built-in function procrustes [53] solves for the parameters $s, a, b, c, d, t_{w}$, and $t_{y}$, given the corresponding pair of control points $x_{i}=\left[x_{i}, y_{i}\right]$ and $x_{i}^{\prime}=\left\{x_{i}^{\prime}, y_{i}^{\prime}\right\}$.

\section{Image Warping}

Suppose we wish to warp an image $I$ so that the set of $n$ control points $\boldsymbol{x}_{i}=\left\{x_{2}, y_{l}\right\}$ are mapped to new positions, $\boldsymbol{x}_{i}^{\prime}-\left\{x_{i}^{\prime}, y_{i}^{l}\right\}$. We again require a continuous vector-valued mapping function, $f$, such that $f\left(x_{i}\right)=x_{i}^{\prime}$. Given such a function, we can project each pixel of image $\boldsymbol{I}$ to a new image $\boldsymbol{I}$. However, in practice, it is better to find the reverse map $\boldsymbol{f}^{\prime}$ (reverse-mapping), taking the control points $\boldsymbol{x}_{\boldsymbol{i}}^{\prime}$ to $\boldsymbol{x}_{\boldsymbol{i}}$, in order to avoid 
interpolation problems. For each pixel in the target warped image $\boldsymbol{I}^{\mathbf{l}}$, we can determine where it came from $\boldsymbol{I}$ and fill it in. Implementation of image warping forward-map is difficult as two problems may occur: (a) many source pixels can map to the same destination pixel and (b) some destination pixels may not be even covered. However, with reverse-map image warping, since iteration over the destination image is performed, there is no possibility that the problems existing with the forward-map warping, will occur.

\subsection{Summary}

This chapter covered the basic image analysis tools which have been used in this thesis. In particular, the Farag-Abdelmunim Algorithm [37] for image segmentation is used for segmentation of the MRI data to isolate the corpus callosum, and the Algorithm for shape registration [38]. The chapter covered the basic mathematics involved in registration using the ICP algorithm and the Mutual Information (MI) algorithm. The MI algorithm would be value for registering normal to autistic (or vice versa) scans for purpose of normalization and comparison between intensity variations (especially at the

cortical surface). The shape modeling approaches of Abdelmunim and and Farag [38] and Fahmi and Farag [40] are of value for both automatic segmentation of the corpus callosum as well as modeling the contours of the corpus callosum. 


\section{CHAPTER 3}

\section{SHAPE REPRESENTATION}

\subsection{Introduction}

An object's shape is what is invariant to rotation, translation and scaling; that is, all the geometrical information that remains when location, scale, and rotation effects are filtered out from an object (e.g., [54]). Stated another way, the Euclidean similarity transformations of scaling, translation, and rotation have an invariant effect on shape, and if two objects can be mapped to one another using these transformations, then they are said to have the same geometric shape."

Shapes can be divided into two categories: Static or Dynamic. Static Shapes are rigid shapes that do not change in time by deformation or articulation, for example, a model of a ship. Dynamic shapes are rigid shapes that do change in time by deformation or articulation. An MRI image of a human brain can be thought of as static, however the changes in the brain that occur over the course of many years from growth and disease can be thought of as Dynamic. The human face is a dynamic shape due to effects of gestures, talking and expressions. A fluid mechanic model resembling the state of a weather phenomenon is dynamic, etc.

Shape representation is an important aspect of modern computational geometry and refers to the general framework that describes shape information, including data representation, meshing, surface fitting and convex hulls. Shapes are typically defined 
as an object, an abstraction, or a representation. An object can be physically dealt with (seen, touched) such as a ball. An abstraction is a quality separated from an actual example, such as the concept of a ball being a sphere. A representation is the way a shape is symbolized; for example, representing the ball as an equation for a sphere $\left(\mathrm{r}^{2}=\right.$ $\left.x^{2}+y^{2}+z^{2}\right)$

Shape Representation takes many forms in the sense that it may only describe a particular global feature of shape, such as the boundary that surrounds an object (Boundary-based representations like curves and surfaces), the region that is occupied by an object (Region-based representation like images/volumes), or by the mathematical coefficients that describe a transform-domain (Transform-based representation such as Fourier Descriptors and Spherical harmonics). Each representation incorporates certain aspects of a shape. In general, shape representation approaches may be divided into two broad categories: Data-based representations and Model based representations. Databased representations include point-based, landmark-based, boundary-based, and volumebased representations. Model-based representations include global feature-based and graph-based Representations [54][55].

\section{Point based Representations:}

Point-based representations render a shape in terms of a point cloud or range images. While they may provide a useful visual display, they generally lack the structural information of the shape such as its connectivity. Multiple Range images of an object (usually acquired via a laser scanner) are used to reconstruct a 3D model. Range 
Images are acquired by moving an object through a continuous sheet of last light, which gets observed by a camera tuned to that wavelength of light. The scanner measures the depth of the object and places it on a position of a grid in a range images.

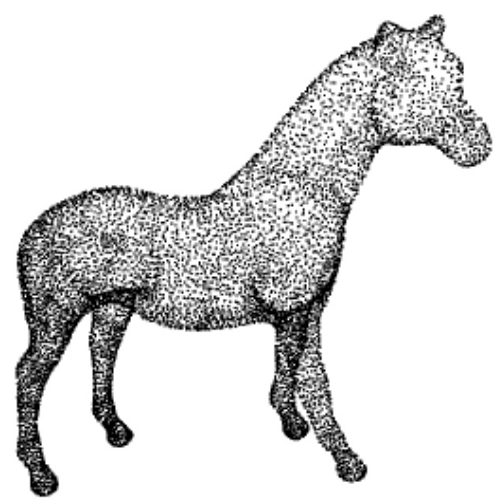

Figure 12: An example of point-based respresentation of a shape.

\section{Landmark-based Representations:}

Landmark-based representations describe a shape boundary by a set of specific points. As defined by Dryden and Mardia, "A Landmark is a point of correspondence on each object/shape which matches between and within populations" [55]. They further divide landmarks into three subgroups (Anatomical, Mathematical, and Pseudo). Anatomical landmarks are points that correspond between organisms biologically as defined by an expert (e.g., [56]). Commonly used synonyms for landmarks are nodes, vertices, homologous points, etc.

\section{Boundary Based Representations}

Boundary Based representations, commonly used in CAD and graphics, represent an object in terms of its boundary or surface. This type of representation is one of the 
two main representations used for this thesis. Boundary data is defined in terms of polygonal meshes, parametric forms, and implicit surfaces. Figure 13 shows a onedimensional example of an open boundary; the more poly-lines (line segments sharing endpoints) used, the better the approximation.

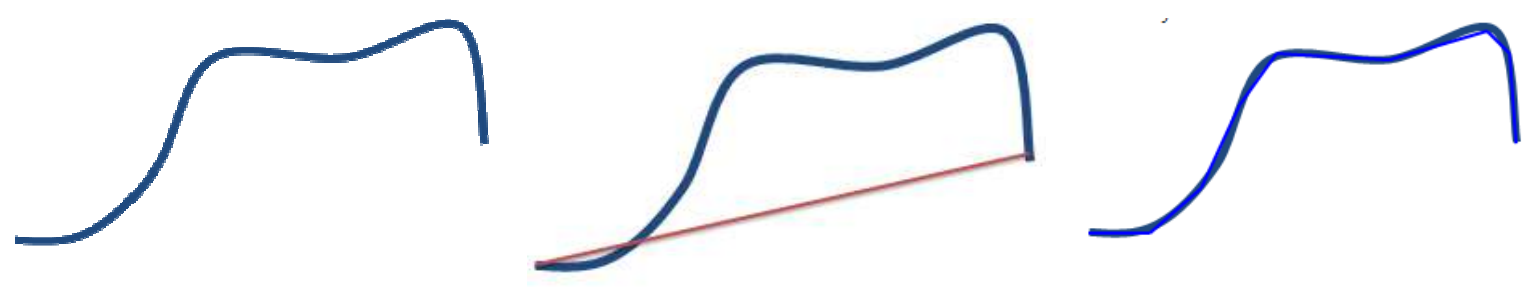

Figure 13: Boundary representation and approximation with single (middle) and multiple lines (rightmost).

\section{Curves:}

Curves in the standard 2-dimensional Euclidean space $\mathbb{R}^{2}$ are called plane curves which can be described either in an explicit form, $y=f(x)$; for example, as a function graph, or implicitly as a set of points which specify an equation $\mathbf{f}(\mathrm{x}, \mathrm{y})=0$; this equation can be represented in a parametric form as $\mathrm{x}(\mathrm{t})=(\mathrm{x}(\mathrm{t}) \mathrm{y}(\mathrm{t}))^{\mathrm{T}}$, however we need to place restrictions on $\mathbf{f}$ such that the solution of $\mathrm{f}(\mathrm{x}, \mathrm{y})=0$ does not fill the entire plane.

Algebraic curves are curves defined by $f$ such that $f$ is a polynomial function in two variables. Equations of the first degree define straight lines, while equations of the second degree define ellipses, parabolas or hyperbolas. Curves in the standard 3- 
dimensional Euclidean space $\mathbb{R}^{3}$ are called space curves which can be defined as intersections of two surfaces defined implicitly by $f(x, y, z)=0$, and $g(x, y, z)=0$.

Definition: Let $\left\{\mathrm{x}_{1}, \mathrm{x}_{2}, \ldots, \mathrm{x}_{\mathrm{n}}\right\}$ be a set of points in the d-dimensional Euclidean space, a curve can be defined in terms of these points as:

$$
\text { c: } x(t)=f_{1}(t) x_{1}+f_{2}(t) x_{2}+\cdots+f_{n}(t) x_{n}=\sum_{k=1}^{n} f_{k}(t) x_{k}
$$

Where $\mathrm{f}_{\mathrm{k}}(\mathrm{t})$ are continuous functions defined on the intervalt $\in[0,1]$. The points $\mathrm{x}_{1}, \mathrm{x}_{2}, \ldots, \mathrm{x}_{\mathrm{n}}$ are called control point and $\mathrm{f}_{\mathrm{k}}(\mathrm{t})$ are called the basis functions of the curve $c$. Next we will discuss one of the more popular curves and how they may be used to interpolate points.

In this thesis, we will focus on curve representations, specifically, Bezier curves/patches and the Fourier descriptions. Therefore, these two representations will be studied in details in the rest of this chapter.

\subsection{Bézier Curves}

Bézier curves are one of the most popular representations for curves.

Definition 3.1: Let $\mathrm{x}_{0}, \mathrm{x}_{1}, \mathrm{x}_{2}, \ldots, \mathrm{x}_{\mathrm{n}}$ be a set of control points (or vertices of a shape), a Bézier curve of degree $\mathrm{n}$ is given by:

$$
\mathrm{x}(\mathrm{t})=\sum_{\mathrm{k}=0}^{\mathrm{n}} \mathrm{B}_{\mathrm{k}}^{\mathrm{n}}(\mathrm{t}) \mathrm{x}_{\mathrm{k}} \quad \mathrm{t} \in[0,1]
$$

Where the basis functions $\mathbf{B}_{k}^{\mathrm{n}}(t)$ are the Bernstein polynomials defined by; 


$$
B_{k}^{n}(t)=\left(\begin{array}{l}
n \\
k
\end{array}\right) t^{k}(1-t)^{n-k}
$$

Where $\left(\begin{array}{l}\mathrm{n} \\ \mathrm{k}\end{array}\right)=\frac{\mathrm{n} !}{\mathrm{kn} !(\mathrm{n}-\mathrm{k}) !}$

Bézier curves interpolate the end points $\mathrm{x}_{0}$ and $\mathrm{x}_{\mathrm{n}}$, that is it connects the end points in a fashion directed by in-between control points, which do not lie on the curve, this is called endpoint interpolation property.

Definition 3.2: Given two control points $\mathrm{x}_{0}, \mathrm{x}_{1}$, a Linear Bézier curve is simply a straight line between those two points, the curve is given by:

$$
x(t)=(1-t) x_{0}+t x_{1} \quad, \quad t \in[0,1]
$$

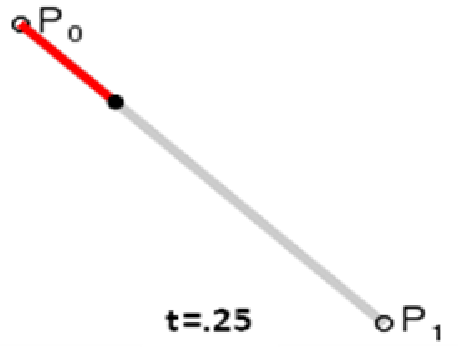

Figure 14: The tin the function for a linear Bézier curve can be thought of as describing how far $x(t)$ is from $P 0$ to P1. For example when $t=0.25, x(t)$ is one quarter of the way from point P0 to P1. As $t$ varies from 0 to $1, x(t)$ describes a curved line from $P 0$ to $P 1$.

Definition 3.3: A quadratic Bézier curve is the path traced by the function $\mathbf{x}(\mathbf{t})$ given three control points $\mathrm{x}_{0}, \mathrm{x}_{1}, \mathrm{x}_{2}$ :

$$
x(t)=(1-t)^{2} x_{0}+2 t(1-t) x_{1}+t^{2} x_{2}, t \in[0,1]
$$

We note, a quadratic Bézier curve is also a parabolic segment. 


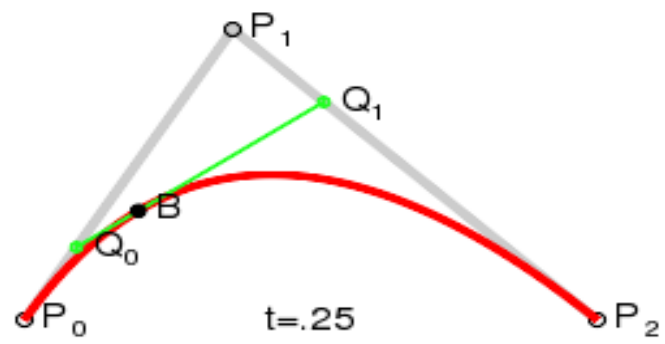

Figure 15: For quadratic Bézier curves one can construct intermediate points $Q 0$ and $Q 1$ such that as $t$ varies from 0 to 1 : Point $Q 0$ varies from $P 0$ to $P 1$ and describes a linear Bézier curve. Point $Q 1$ varies from P1 to P2 and describes a linear Bézier curve. Point $x(t)$ varies from $Q 0$ to $Q 1$ and describes a quadratic Bézier curve.

Definition 3.4: Four control points $\mathrm{x}_{0}, \mathrm{x}_{1}, \mathrm{x}_{2}, \mathrm{x}_{3}$ in the plane or in three-dimensional space define a cubic Bézier curve, the curve starts at $\mathrm{x}_{0}$ going toward $\mathrm{x}_{1}$ and arrives at $\mathrm{x}_{3}$ coming from the direction of $\mathrm{x}_{2}$, usually it will not pass through $\mathrm{x}_{1}$ or $\mathrm{x}_{2}$, these points are only there to provide directional information, the parametric form of the curve is:

$$
\begin{gathered}
x(t)=\left(1-3 t+3 t^{2}-t^{3}\right) x_{0}+\left(3 t-6 t^{2}+3 t^{3}\right) x_{1}+\left(3 t^{2}-3 t^{3}\right) x_{2}+t^{3} x_{3}, t \\
\in[0,1]
\end{gathered}
$$

Definition 3.5: Let $\mathrm{x}_{0}, \mathrm{x}_{1}, \mathrm{x}_{2}, \ldots, \mathrm{x}_{\mathrm{n}}$ be a set of control points, a Bézier curve of degree $\mathrm{n}$ given by:

$$
\mathrm{x}(\mathrm{t})=\sum_{\mathrm{k}=0}^{\mathrm{n}} \mathrm{B}_{\mathrm{k}}^{\mathrm{n}}(\mathrm{t}) \mathrm{x}_{\mathrm{k}} \quad \mathrm{t} \in[0,1]
$$

can be expressed recursively as follows:

Let $\mathrm{x}_{\mathrm{x}_{0} \mathrm{x}_{1} \ldots \mathrm{x}_{\mathrm{n}}}(\mathrm{t})$ denote the Bézier curve denoted by the control pointsx $\mathrm{x}_{0}, \mathrm{x}_{1}, \mathrm{x}_{2}, \ldots, \mathrm{x}_{\mathrm{n}}$, then,

$$
x(t)=x_{x_{0} x_{1} \ldots x_{11}}(t)=(1-t) x_{x_{0} x_{1} \ldots x_{11}-1}(t)+t x_{x_{1} x_{2} \ldots x_{11}}(t)
$$




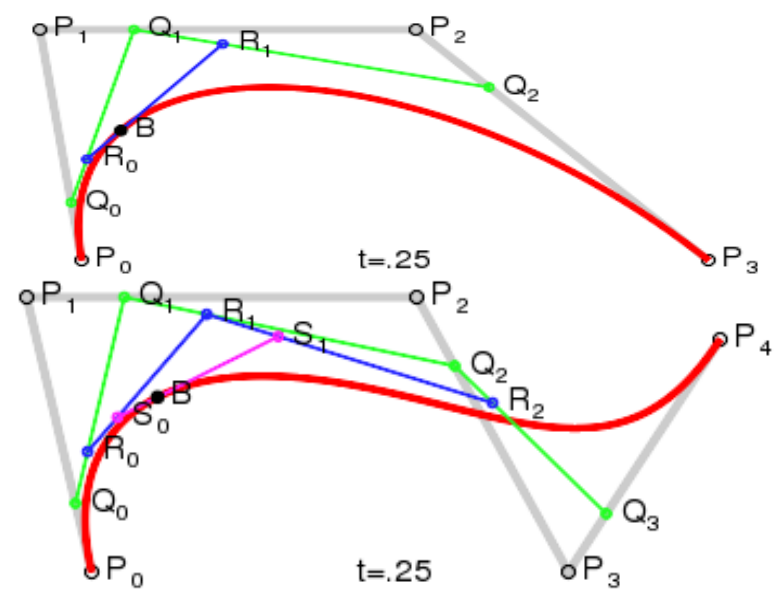

Figure 16: For higher-order curves one needs correspondingly more intermediate points. For cubic curves one can construct intermediate points Q0, Q1 \& Q2 that describe linear Bézier curves, and points $R 0 \& R 1$ that describe quadratic Bézier curves. For fourth-order curves one can construct intermediate points $Q 0, Q 1, Q 2$ \& $Q 3$ that describe linear Bézier curves, points $R 0, R 1 \& R 2$ that describe quadratic Bézier curves, and points $S 0 \& S 1$ that describe cubic Bézier curves.

Hence, the Bézier curve of degree $n$ is a linear interpolation between two Bézier curves of degree $n-1$.

Definition 3.6: Let $\mathrm{x}_{0}, \mathrm{x}_{1}, \mathrm{x}_{2}, \ldots, \mathrm{x}_{\mathrm{n}}$ be a set of control points of the Bézier curve, the polygon formed by connecting the Bézier points with lines, starting with $\mathrm{x}_{0}$ and finishing with $\mathrm{x}_{\mathrm{n}}$, is called the Bézier polygon. The convex hull of the Bézier polygon contains the Bézier curve.

\subsubsection{The Bernstein polynomials}

The Bernstein polynomials are a partition of unity, i.e. $\sum_{k=0}^{n} B_{k}^{n}(t)=1$, hence

Bézier curves are affine invariant. This is a direct application of the binomial theorem;

$$
\sum_{k=0}^{n} B_{k}^{n}(t)=\sum_{k=0}^{n}\left(\begin{array}{l}
n \\
k
\end{array}\right) t^{k}(1-t)^{n-k}=(t+(1-t))^{n}=1
$$


Definition 3.7: The Bernstein polynomials $B_{k}^{n}(t)$ are defined such that $0 \leq B_{k}^{n}(t) \leq 1$ for $t \subset[0,1]$.

A point of the Bézier curve $\boldsymbol{x}(t)-\sum_{k=0}^{n} B_{k}^{n}(t) \mathbf{x}_{\mathrm{kn}}$ is thus a weighted average of the points $\mathbf{x}_{0}, \mathbf{x}_{1}, \mathbf{x}_{2}, \ldots, \mathbf{x}_{n}$.

Definition 3.8: The convex hull of the curve $\mathbf{x}(t)$ is the set of all weighted averages of $\mathbf{x}_{n}, \mathbf{x}_{1}, \mathbf{x}_{2,}, \ldots, \mathbf{x}_{n}$. The Bézier curve thus lies in the convex hull of the points $\mathbf{x}_{0}, \mathbf{x}_{1}, \mathbf{x}_{2}, \ldots, \mathbf{x}_{n}$, where the convex hull is defined by a polygon created from these points.

While 2D objects require groups of line segments (polygons) for shape representation, 3D objects need all their surfaces represented. Using data such as vertices, edges, and faces, polygons are represented. Polygons are made by many curves unified to make a closed curve. Indented polygons are called concave, while polygons with no indentation are referred to as convex, Figure 17.
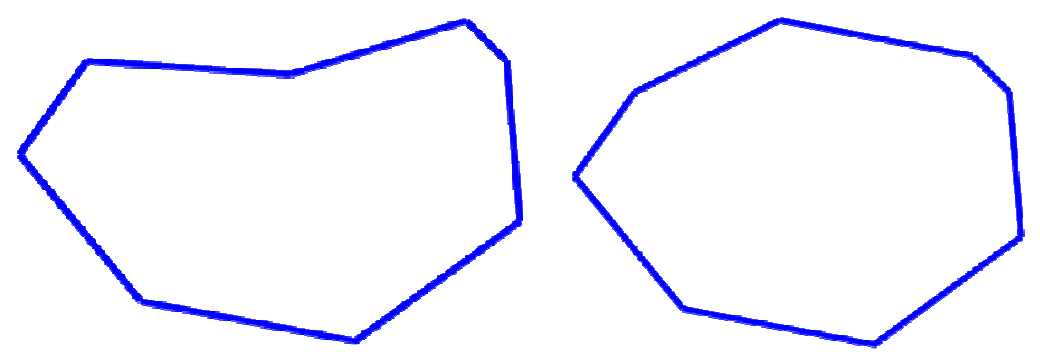

Figure 17: Illustration of Concave (left) and convex (right) polygons. 

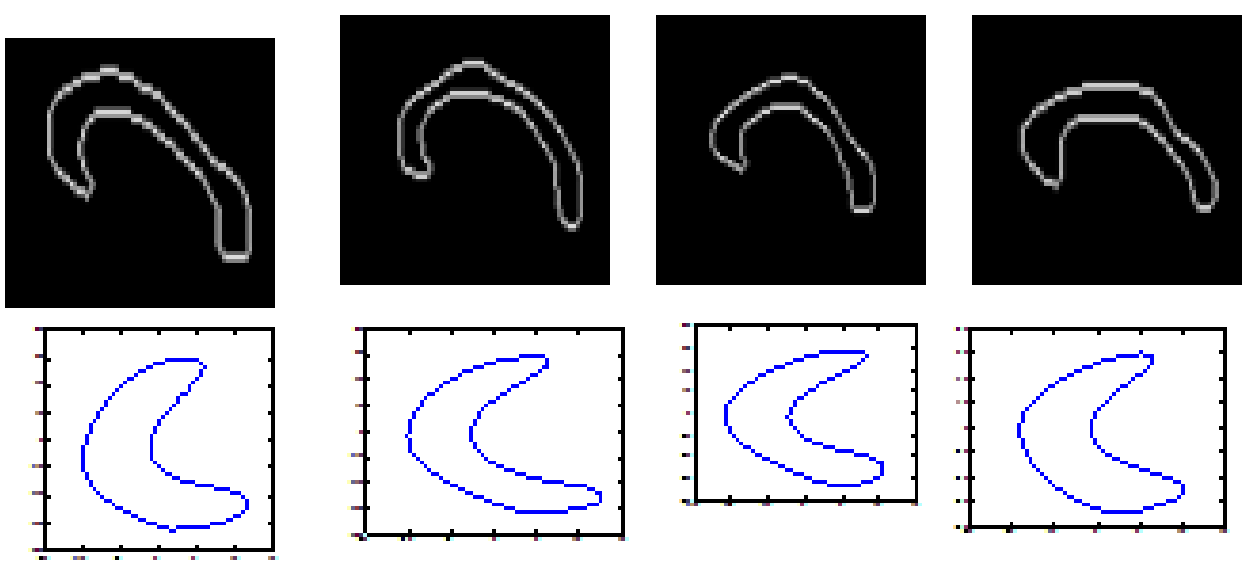

Figure 18: Examples of Bezier curves fitting on the corpus callosum using the Bernstein polynomials of $50^{\text {th }}$ order. The fittings are not aligned with the original contours.

\subsubsection{Surface Mesh}

Polygons may be assembled together in groups to form surfaces called meshes. Surface modeling is usually represented by polygon meshes. Constructing a mesh is a major component in the modeling and analysis of shapes, especially in 2D and 3D. Even though quantification of changes in autistic vs. normal brains are best studied in 3D (thus requiring a meshing process for the 3D segmented volumes from MRI scans) our focus in this thesis is limited to 1D closed contours representing the Corpus Callosum; hence, the issue of meshing will not studied further.

\subsection{Model-based Representation}

Model based representation of an object describes certain global or topological features included in the shape. Global feature based methods include the global properties such as moments, distance vs. angle, Fourier Descriptors and spherical 
harmonics. This thesis explores Fourier Descriptors in more detail. Fourier Descriptors are a very powerful tool because, unlike other techniques, they are invariant to scaling, translation, and rotation because of their basis using the Fourier Transform.

\subsubsection{Fourier Transformation}

The Fourier transform (FT) is an operator which transforms a real variable, or function, in the time domain into another variable in the frequency domain. Hence, the Fourier transform if often called the frequency domain representation of the original function. This representation is fundamental in calculus and has been the basis of major developments in the theory and applications of signals and systems for over two centuries, and an enormous body of literature exists since its invention by Joseph Fourier in the turn of the $19^{\text {th }}$ century (e.g., [58]). We will barely touch some of the basics of the Fourier transform in this chapter and focus on an aspect of it that has been very useful in shape analysis, namely the Fourier Descriptors.

Let $x(t)$ be a complex valued function of a real variable in the time domain. In the continuous time, the Fourier transform of this function can be written as:

$$
X(f)=\mathrm{F}[\mathrm{x}(\mathrm{t})]=\int_{-\infty}^{\infty} x(t) \exp (-2 \pi j f t) d t
$$

The inverse Fourier transform can be described as

$$
x(t)=\mathrm{F}^{-1}[\mathrm{x}(\mathrm{t})] \int_{-\infty}^{\infty} x(f) \exp (2 \pi j f t) d f
$$


In the Discrete Fourier transform (DFT) the function are discrete variables. Let $\left\{x_{0}, x_{1}, \ldots, x_{N-1}\right\}$ is complex-valued function of real variables. The DFT of these functions can be represented as

$$
X_{f}=\sum_{n=0}^{N-1} x_{n} \exp \left(-\frac{2 \pi j}{N} f n\right)
$$

The inverse DFT can be written as

$$
x_{n}=\frac{1}{N} \sum_{f=0}^{N-1} X_{f} \exp \left(\frac{2 \pi j}{N} f n\right)
$$

In the next section, we define the Fourier Descriptors which is used in shape representation and many applications.

\subsubsection{Fourier Descriptor}

Fourier descriptors are representation of vertices of a polygon in terms of the basis functions of the discrete Fourier transform. It has been used for various applications of pattern recognition for over four decades (e.g., [59]-[63]). Let $\boldsymbol{N}$ represents the number of points of a given shape. The initial points can be represented as $\mathbf{z}$. The Fourier descriptors $(\{\mathbf{c}(\mathbf{k}) ; k=M / \mathbf{2} \| \mathbf{1}, \ldots, M / 2\})$ are the coefficients of the Fourier transform of input points $\{(\mathbf{z}(\mathbf{i}) ; i=0, \ldots, N-1)\}$. The variables of the function can be as below.

- $\quad N$ : The number of points of a given shape, (input).

- $\quad \boldsymbol{M}$ : The number of the Fourier descriptors you want to use (user selection).

- $\quad \boldsymbol{L}$ : The number of points needed to be reconstructed. 
- $\tilde{\boldsymbol{z}}_{\ell}$ : The reconstructed points where $\ell=\{0, \ldots, t,-1\}$.

- $z_{i}$ : The given (original) points where $i=\{0, \ldots, N-1\}$.

- $c_{k}$ : The Fourier descriptors where $\mathrm{k}=\{-M / 2+\mathbf{1}, \ldots, M / 2\}$.

For a given closed contour, the points represented as $z(i)=x(i)+j \cdot y(i)$, where $\{i=0, \ldots, N-1\}$, the points should be selected in a clockwise or counterclockwise direction [61] as shown in Figure 19. From the start and to the end point, we encounter the coordinate pairs $\left(x_{0}, y_{0}\right),\left(x_{1}, y_{1}\right), \ldots,\left(x_{N-1}, y_{N-1}\right)[63]$.

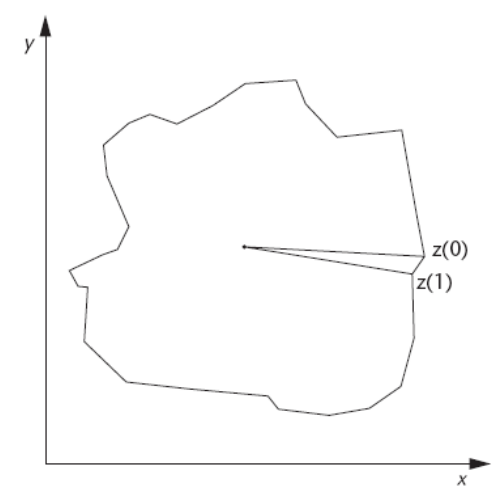

Figure 19: Point selection from a given closed contour (the image is adopted from [63]).

The Fourier descriptor can be defined as

$$
c_{k}=\frac{1}{N} \sum_{i=0}^{N-1} z_{i} \exp \left(-j 2 \pi \frac{i k}{N}\right) \quad \text { where } \quad \mathrm{k}=\{-M / 2+1, \ldots, M / 2\}
$$

The reconstructed points are calculated as

$$
\tilde{z}_{\ell}=\sum_{k=-\frac{M}{2}+1}^{\frac{M}{2}} c_{k} \exp \left(j 2 \pi \frac{\ell k}{N}\right) \quad \text { where } \ell=\left\{0, \ldots, I_{L}-1\right\}
$$

The number of the Fourier descriptor is not dependent on the number of the given and reconstructed points. It is related with how much information we want to reconstruct 
from the given points. In the Fourier descriptor representation, the $c_{k}$ coefficients around $c_{D}$ (DC term) describe the low-frequency information. The high frequency information is carried in the coefficients around $c_{-M / 2}$ and $c_{M / 2}$ terms. The Fourier descriptors at lower frequency represent the general shape, whereas the Fourier descriptors at higher frequency describe the details and sharp corners [59- 62].

\section{Example 1: Shape representation of the corpus callosum}

In this experiment, we are given a closed contour of corpus callosum. Our aim is to obtain a shape representation using various number of the Fourier descriptor. When we begin with a low number of the Fourier descriptor, we obtained a general shape of the input contour. The more Fourier descriptor we use, the more details of the given shape we obtain. Because the $c_{k}$ coefficients around $c_{0}$ (DC term) describe the low-frequency information, the descriptors at low frequency contain the general information of the input data whereas the descriptors at high frequency contain the details. Figure 20 shows the results of the shape representation experiment. The variables of this experiment are shown below.

- $\quad N$ : The number of points of a given shape, (input) $=416$

- $\quad M$ : The number of the Fourier descriptors used, e.g., 2, 4.. 100.

- $\quad L$ : The number of points reconstructed $=416$. 


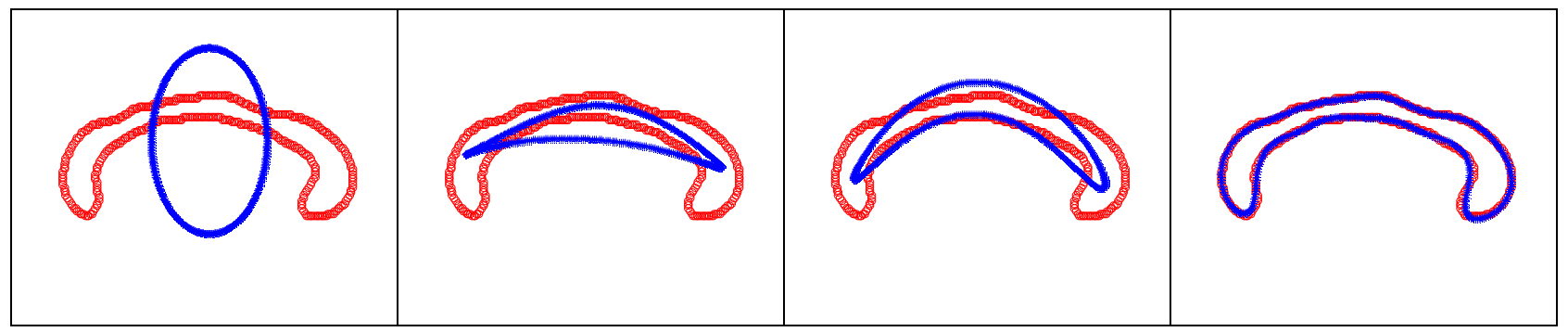

Figure 20: Shape representation of the corpus callosum. Red and blue points represent the original contour and reconstructed contours. Reconstructions using 2, 4, 6 and 14 Fourier descriptors from left to right, respectively.

Hence, the Fourier Descriptors may be used as the feature describing the shape. We shall show in Chapter 4 that the statistics of these descriptors provides discrimination between the autistic and normal subjects.

\section{Example 2: Use of Fourier Descriptors for Interpolation}

In this experiment, we are given a contour of corpus callosum which has some missing points. Our aim is to interpolate the missing points. The interpolated points are not perfect but it is very close to the original shape. In this experiment, we change the number of the Fourier descriptors, too. When the number of descriptors are high enough the closest contour is obtained as shown in the Figure 21. The variables of this experiment are shown below.

- $\quad N$ : The number of points of a given shape, (input) $=350$

- $\quad M$ : The number of the Fourier descriptors you want to use $=$ $\{2,4, \ldots, 30\}$.

- $\quad L$ : The number of points needed to be reconstructed $=416$ (can be any number). 


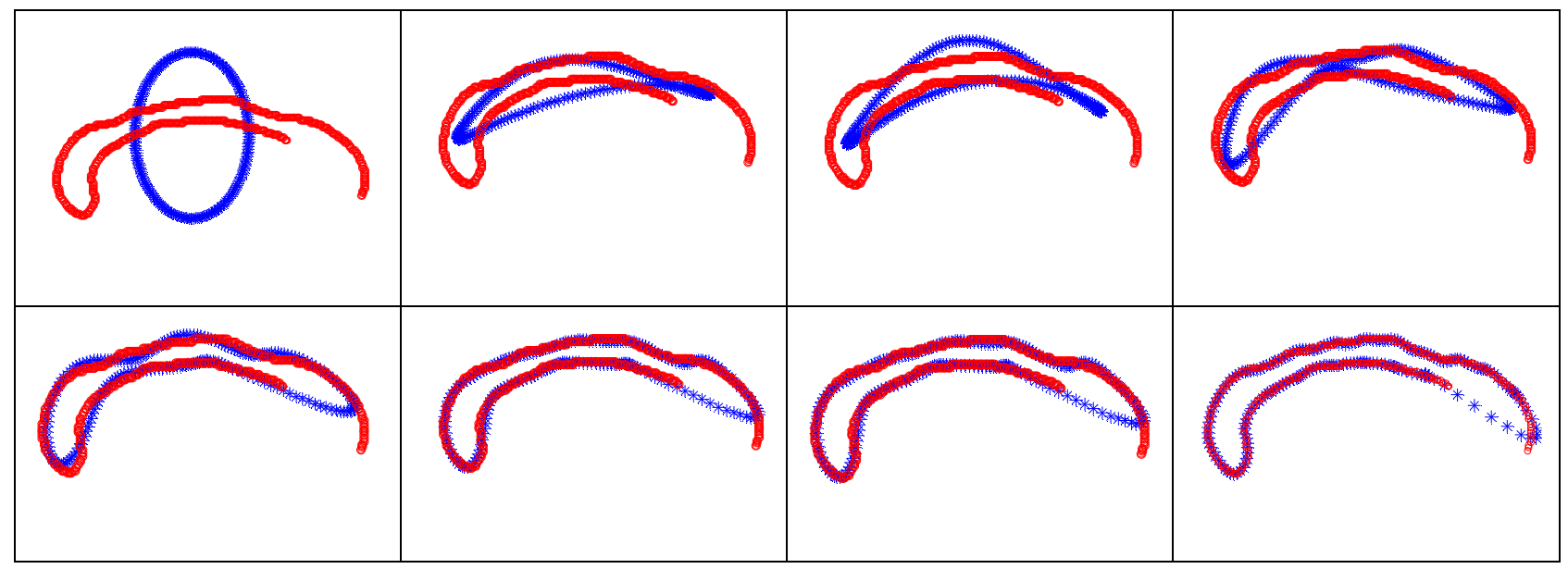

Figure 21: Interpolation of the corpus callosum shape. Red and blue points represent the given contour (with missing points) and reconstructed contours, respectively. From left to right on both rows reconstructions using 2, 4,8,10,14,18,24, and 30 Fourier descriptors, respectively.

Figure 22 shows other interpolation results of more difficult cases with missing data.
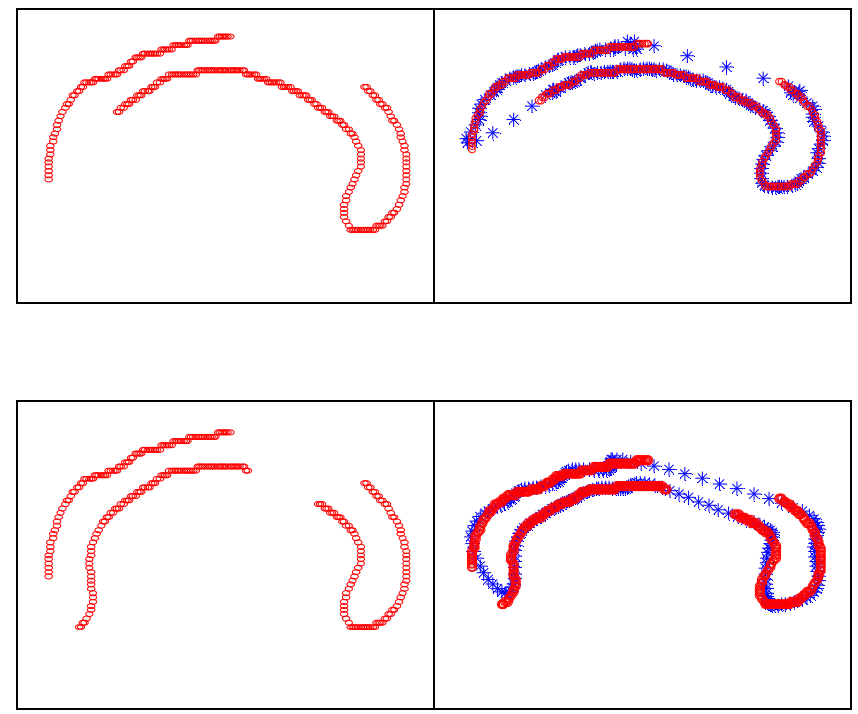

Figure 22: Other interpolation results of more difficult cases. Red shows the initial points and blue shows interpolation results using 60 Fourier descriptors. 
The above examples illustrate the power of the Fourier Descriptors approach for contour analysis. First report for its use with the corpus callosum as well as closed contours of inner parcelation of the White Matter of the human brain was in the CVIP Lab [64][65]. We extend this work further as described in Chapter 4.

\subsection{Summary}

In this chapter, we studied various approaches for shape representation. The terminologies of polygons were introduced and two approaches were studied in details for contour modeling, Bezier curves and the Fourier Descriptors. Both approaches form the basis of the experimentation on the real data used in this thesis. 


\section{CHAPTER 4}

\section{SHAPE ANALYSIS OF THE CORPUS CALLSOUM}

\subsection{Introduction}

In this chapter we examine the effectiveness of the shape modeling approaches in Chapter 3 for the analysis of the corpus callosum. The Fourier Descriptors (FD) approach is invariant to translation, rotation and scaling; thus it is of immediate value for shape analysis. We will investigate the global changes among normal and autistic populations using the FD coefficients, and also the power spectrum. We may extract this information from the raw contours of the two populations, and we may also use the globally aligned contours. The Bezier Patches approach is a parametric approach for connecting the vertices of a shape. The Bernstein polynomials are used in the fitting process. We have experimented with polynomials up to the $50^{\text {th }}$ order. The histograms of these coefficients are examined and used for classification. We will highlight these techniques after we describe the data which has been used to test the research in this thesis.

\subsection{Experimental MRI Data Set}

The postmortem MRI data used in this study is described in detail in [30]. In order to optimize white-gray matter substance contrast in formalin-fixed brains, a proton density weighted imaging sequence was used. The method employed a 1.5 Tesla GE MRI system to scan brains that have been placed within a special device that avoids dehydration during the scanning procedure. The scan time lasted an average of 50 
minutes, 29 seconds. A Fast Spin Echo (FSE) technique with a long Repetition time (TR) of $6700 \mathrm{~ms}$ and a short Echo time (TE) of $8.23 \mathrm{~ms}$ and an Echo train of 4, render coronal images with excellent gray-white matter contrast. The technique allows the full coverage of brains with 114 slices, $1.6 \mathrm{~mm}$ in thickness, with no inter-slice gap. A high resolution K-space data set consisting of 256 points in the phase and Zero-fill interpolation (ZIP) 512 points in the frequency of both encode direction [30]. An in-plane resolution of 625 microns X 312.5 microns, a field of view of $16 \mathrm{~cm} \times 16 \mathrm{~cm}$, and a number of repeated excitations (NEX) of 7 with a bandwidth of 62.5 were used. These techniques demonstrate good results in samples that have been fixed for 6 weeks to 15 months (Schumann et al., 2001). All images were acquired with the same 1.5-T Sigma MRI scanner (General Electric, Milwaukee, Wisconsin) using a 3-D spoiled gradient recall acquisition in the steady state (time to echo, 5 milliseconds; time to repeat, 24 milliseconds; flip angle, 450; repetition, 1; field of view, $24 \mathrm{~cm} 2$ ). Contiguous axial slices, $1.5 \mathrm{~mm}$ thickness (124 per brain), were obtained. The images were collected in a 192x256 acquisition matrix and were 0-filled in k space to yield an image of 256x256 pixels, resulting in an effective voxel resolution of approximately $1.0 \times 1.0 \times 1.5 \mathrm{~mm}[65]$. Eight whole brains and six hemispheres coronal volumes of size $512 \times 512 \times 114$ were used. Each slice is $1.6 \mathrm{~mm}$ thick with an in-plane resolution of $0.625 \times 0.3125 \mathrm{~mm}$, on which the WM appears dark, the GM appears light, and the fluid appears brighter. Due to different factors including the removal of the brain from the skull and fixation problems, distortions such as large deep cuts, commonly occur and are revealed in the MRI scans. 
In the experiments in this thesis we use the contours of the corpus callosum of 22 autistic and 16 normal subjects from the sagittal plane cross sections of registered brains (i.e., nearly same anatomical locations). These contours were obtained by co-registering the normal and autistic scans and using single slices from the registered volumes. Coregistration can be performed by various techniques and contours were generated manually from the slices of the co-registered volumes.

\subsection{Corpus Callosum Representation}

\subsubsection{Bézier Curves}

Corpus Callosum can be represented as a parametric curve which can be defined as follows. Let $\left\{x_{1}, x_{2}, \ldots, x_{M}\right\}$ be a set of points in the 2-dimensional Euclidean space, a corpus Callosum curve can be defined in terms of these points as:

$$
\text { c: } \quad x(t)=f_{0}(t) x_{0}+f_{1}(t) x_{1}+\cdots+f_{M}(t) x_{M}=\sum_{k=0}^{M} f_{k}(t) x_{k}
$$

Where $f_{k}(t)$ are continuous functions defined on the interval $t \in[0,1]$. The points $\mathrm{x}_{1}, \mathrm{x}_{2}, \ldots, \mathrm{x}_{M}$ are called control point and $\mathrm{f}_{\mathrm{k}}(\mathrm{t})$ are called the basis functions of the curve ${ }^{c}$.

Curve generation/reconstruction can be thought of as an interpolation process where the control points are interpolated to generate points whose locus is the curve. The simplest form of interpolation is Lagrange interpolation; however it does not satisfy the convex hull property. Typically, we would like the curve to be more confined, i.e. the area of the convex hull of the curve should not be much greater than the area of the convex hull of the control points. Bézier curves are one of the most popular 
representations for curves which satisfy convex hull property, it can be defined as follows:

$$
\mathbf{x}(t)=\sum_{k=0}^{M} B_{k}^{M}(t) \mathbf{x}_{\mathbf{k}} \quad t \in[0,1]
$$

where the basis functions $B_{k}^{M}(t)$ are the Bernstein polynomials defined as follows:

$$
B_{k}^{M}(t)=\left(\begin{array}{c}
M \\
k
\end{array}\right) t^{k}(1-t)^{M-k}
$$

Bézier curves interpolate the end points $x_{1}$ and $x_{M}$, that is it connects the end points in a fashion directed by in-between control points, which do not lie on the curve, this is called endpoint interpolation property.

Figure 23 shows fitting of corpus callosum for various values of $t$. It is important that the reconstructed curves maintain the main features of the original data. In our case we wanted the Bezier curves to captures the general shape, perimeter/circumference and volume of the original CC contours.
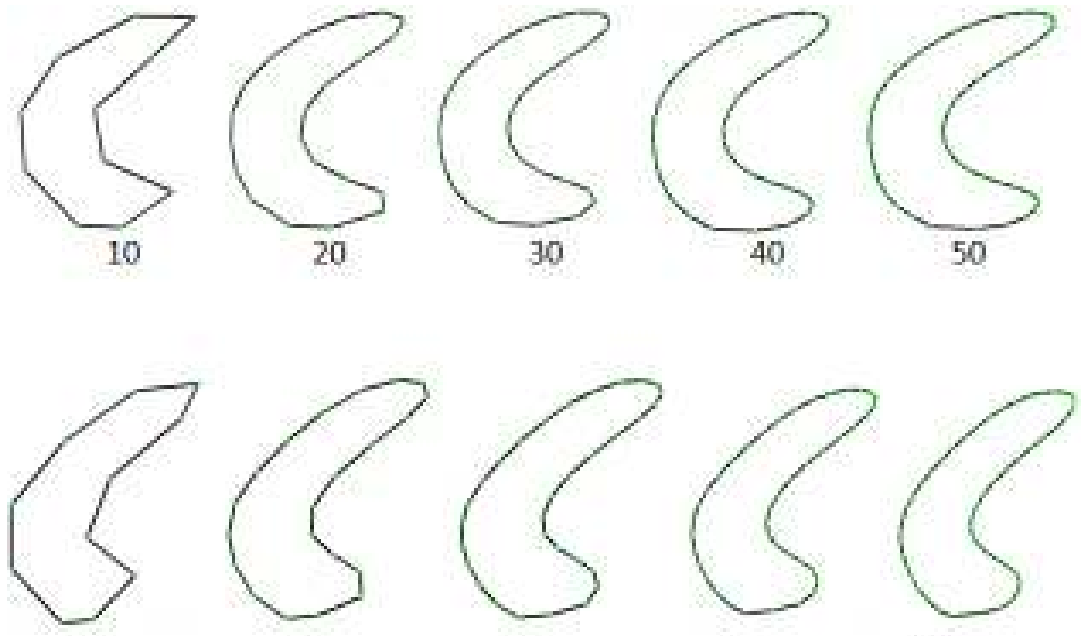

10 20

30

40

50

Figure 23: Fitting a Bezier curve to corpus callosum using different values of the parameter t. Upper (autistic CC) and lower (normal CC). 


\section{Convex Hull:}

It is a useful property to see if a curve lies within its convex hull which can be defined as follows: The convex hull of a corpus Callosum $l$ is the smallest convex set containing $\ell$, hence the convex hull of the set $\mathcal{C}$ is the set of points that are weighted averages of points in that set, thus;

$$
\operatorname{chull}(\mathcal{C})=\left\{\mathbf{x}: \mathbf{x}=\sum_{i=0}^{M} a_{i} \mathbf{x}_{i \bar{l}}, \mathbf{x}_{i} \in \mathcal{c}_{,} \sum_{i=0}^{M} a_{i}=1, a_{i} \geq 0\right\}
$$

Fig 24 shows the convex hulls of Bezier curves with different values of $t$.

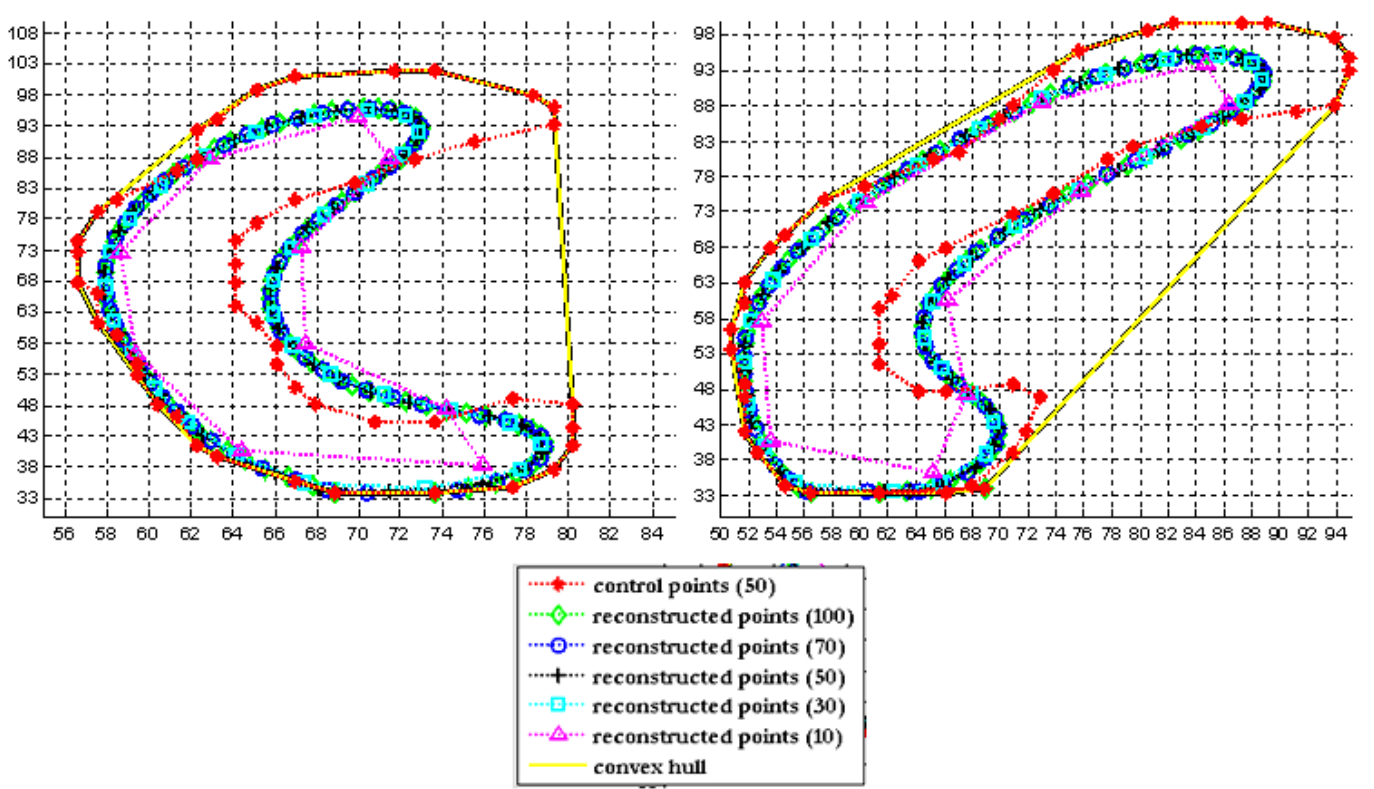

Figure 24: Bézier curve of corpus callosum for autistics (left) and normal subject (right). Control points are shown in red, and the convex hull of the control points is shown in dashed-yellow line. The reconstructed points are obtained by sampling the parameter $t \in|0,1|$ at different resolutions; it is observed that the reconstructed curves are maintained within the convex hull of the control points.

\section{Bezier curves parameterized with respect to $t$}

Equations (2) and (3) can be combined to express the Bezier curve a polynomial (explicit function in the parameter $t$ ) instead of a sum of Bernstein polynomials. Binomial theorem can be applied to the definition of the curve followed by rearrangement to yield, 


$$
\mathbf{x}(t)=\Sigma_{j=v}^{M} c, t^{j} \quad t \in[0,1]
$$

where the coefficients for the expansion of Bernstein polynomials into powers of $t$ are given as follows,

$$
c_{j}=\frac{M !}{(M-j) !} \Sigma_{i=0}^{j} \frac{(-1)^{i} x_{j-i}}{i !(j-i) !}
$$

Proof:

Bezier curve can be defined in terms of Bernstein polynomials as follows,

$$
\mathbf{x}(t)=\sum_{k=0}^{M} B_{k}^{M}(t) \mathbf{x}_{\mathbf{k}}=\sum_{k=0}^{M}\left(\begin{array}{c}
M \\
k
\end{array}\right) t^{k}(1-t)^{M-k} \mathbf{x}_{\mathbf{k}}
$$

According to the Binomial theorem, it is possible to expand any power of $x+y$ into a sum of the form,

$$
(x+y)^{n}=\sum_{i=0}^{n}\left(\begin{array}{l}
n \\
i
\end{array}\right) x^{n-i} y^{i}
$$

Hence we can expand $(1-t)^{M-k}$ as follows;

$$
\begin{aligned}
(1-t)^{M-k}= & (1+(-t))^{M-k}=\sum_{i=0}^{M-k}\left(\begin{array}{c}
M-k \\
i
\end{array}\right)(1)^{M-k-i}(-t)^{i} \\
& =\sum_{i=0}^{M-k}\left(\begin{array}{c}
M-k \\
i
\end{array}\right)(-1)^{i} t^{i}
\end{aligned}
$$

Therefore,

$$
t^{k}(\mathbf{1}-t)^{M-k}=t^{k} \sum_{i=0}^{M-k}\left(\begin{array}{c}
M-k \\
i
\end{array}\right)(-1)^{i} t^{i}=\sum_{i=0}^{M-k}\left(\begin{array}{c}
M-k \\
i
\end{array}\right)(-1)^{i} t^{i+k}
$$

Thus,

$$
\begin{aligned}
\mathbf{x}(t)=\sum_{k=0}^{M}\left(\begin{array}{c}
M \\
k
\end{array}\right)\left(\sum_{i=0}^{M-k}\left(\begin{array}{c}
M-k \\
i
\end{array}\right)(-1)^{i} t^{i+k}\right) \mathbf{x}_{\mathrm{k}} \\
=\sum_{k=0}^{M}\left(\sum_{i=0}^{M-k}\left(\begin{array}{c}
M \\
k
\end{array}\right)\left(\begin{array}{c}
M-k \\
i
\end{array}\right)(-1)^{i} t^{i+k}\right) \mathbf{x}_{\mathbf{k}}
\end{aligned}
$$

where, 


$$
\left(\begin{array}{c}
M \\
k
\end{array}\right)\left(\begin{array}{c}
M-k \\
i
\end{array}\right)=\frac{M !}{k !(M-k) !} \frac{(M-k) !}{i !(M-k-i) !}=\frac{M !}{k ! i !(M-(k+i)) !}
$$

Let $j=k+i \rightarrow k=j-i$,

$$
\left(\begin{array}{c}
M \\
k
\end{array}\right)\left(\begin{array}{c}
M-k \\
i
\end{array}\right)=\frac{M !}{(j-i) ! i !(M-j) !}
$$

Thus,

$$
\begin{aligned}
\mathbf{x}(t)=\sum_{k=0}^{M} & \left(\sum_{i=0}^{M-k}\left(\begin{array}{c}
M \\
k
\end{array}\right)\left(\begin{array}{c}
M-k \\
i
\end{array}\right)(-1)^{i} t^{i+k}\right) \mathbf{x}_{\mathrm{k}} \\
& =\sum_{j=0}^{M}\left(\sum_{i=0}^{M-j-i} \frac{M !}{(j-i) ! i !(M-j) !}(-1)^{i} t^{i+j-i}\right) \mathbf{x}_{j-i} \\
& =\sum_{j=0}^{M}\left(\sum_{i=0}^{M-j-i} \frac{M !}{(j-i) ! i !(M-j) !}(-1)^{i} \mathbf{x}_{j-i}\right) t^{j}
\end{aligned}
$$

from the factorial rules, to evaluate $(j-i)$ ! we need to have $j \geq i$, hence the second summation should be upper bounded by $j$,

$$
\mathbf{x}(t)=\sum_{j=0}^{M} c_{j} t^{j}
$$

where,

$$
c_{j}=\frac{M !}{(M-j) !} \Sigma_{i=0}^{j} \frac{(-1)^{i} x_{j-i}}{i(j-i) !}
$$

Hence, a corpus Callosum can now be represented in terms of the Bézier curve coefficients $c_{m}$ for $m=0,1, \ldots, M-1$. Figure 25 shows the discriminatory power of the coefficients of Eq. (4) where we show the scatter plot of these coefficients for normal and autistic subjects. We can clearly see that the coefficients of the two classes form two distinct clusters. Cluster analysis methods may be used to enhance the discrimination between the two classes (e.g., [49]). 
Next we will use these coefficients as features for designing classifiers.

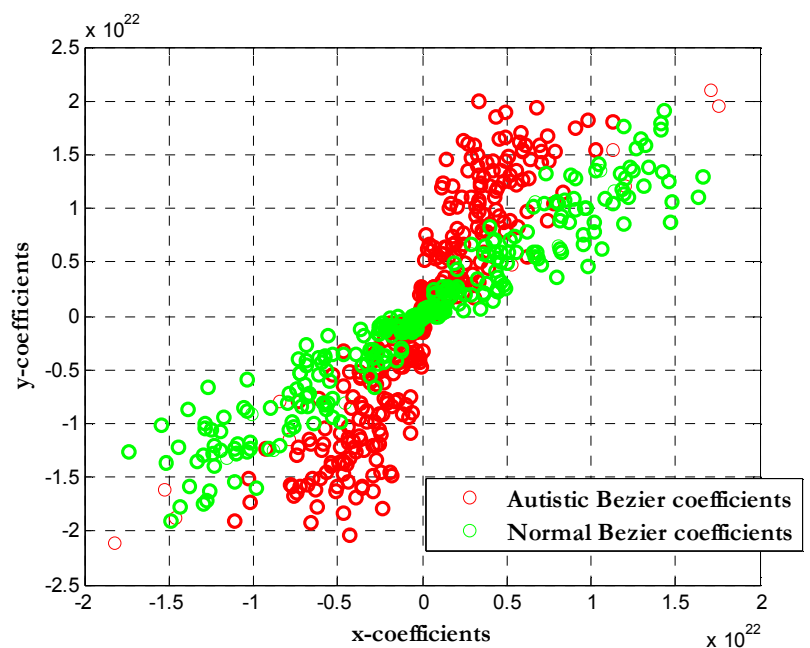

Figure 25: scatter plot of the Bezier coefficients of the corpus callosum of autistic and normal subjects.

\subsubsection{Fourier descriptors}

Fourier descriptor represents the shape in terms of its spatial frequency content. The boundary/contour of a corpus callosum, which can be the control points or the reconstructed points for Bezier curve fitting, is represented as a periodic function which can be expanded using a Fourier series; hence we obtain a set of coefficients which capture the shape information. Let the corpus callosum be represented as a parametric curve $\boldsymbol{c}=\{x(t), y(t)\}$ such that $t \in[0,1]$, by considering a complex image plane, we will end up with a one dimensional function $u(l)-x(L)+j y(L)-u(L+r T)$ for $n=1,2, \ldots$. from which Fourier series expansion is obtained. Figure 20 (Chapter 3) shows examples of reconstructions of the corpus callosum using the Fourier Descriptors method.

In this thesis, the Fourier descriptor of the mean autistic $\overline{\boldsymbol{c}_{A}}$ and the mean normal $\overline{\boldsymbol{c}_{C}}$ corpus callosum are obtained used for classification. 


\subsection{Shape Alignment}

The corpus callosums of normal and autistic subjects need to be put on a common reference in order to exploits the discriminatory features in the two classes. We employed the procrastes technique for shape alignment of the corpus callosum of normal and autistic data (other approaches, e.g., [38][40] would work as well). Figure 26 shows the contours and corresponding Bezier patches for 22 autistic subjects. Figure 27 shows the individual as well as the average contours of these patches.

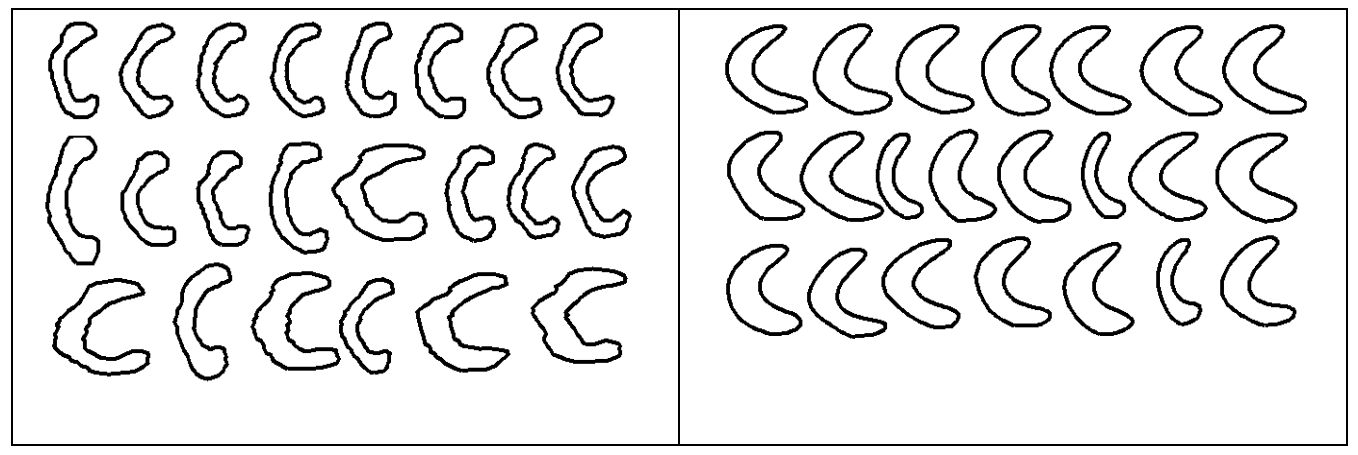

Figure 26: A sample of 22 corpus callosum contours of autistic subjects and their corresponding Bezier curve fitting.

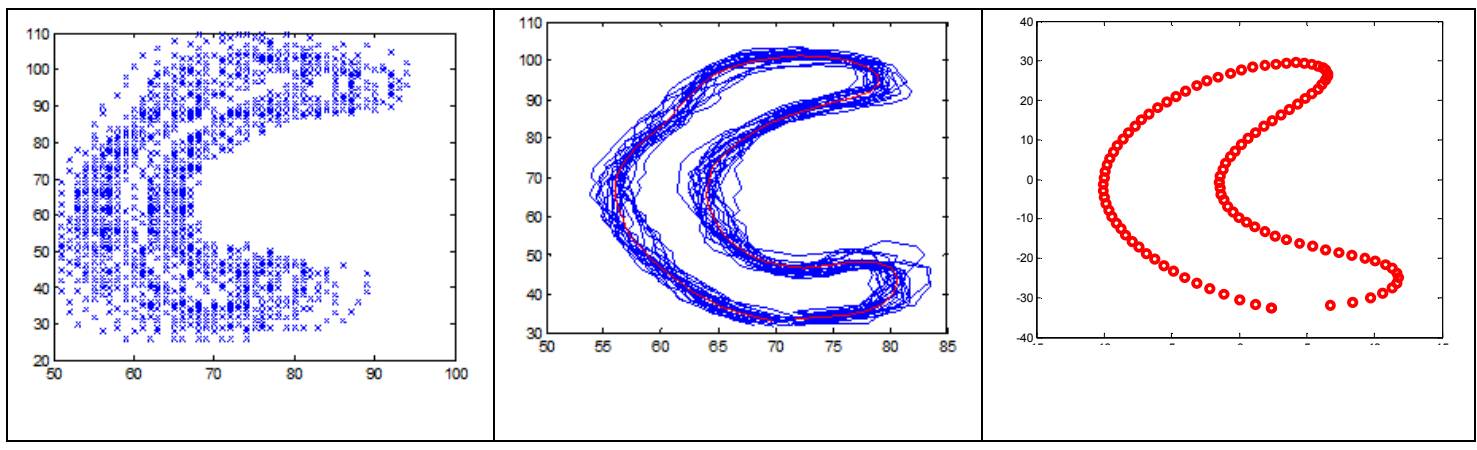

Figure 27: Individual and aligned Bezier contours for 22 autistic subjects and their average shape (red). Left shows row vertices, middle show the contours and left is the mean Bezier contour. 
Figures 28-29 shows the corresponding results for 16 normal subjects in the experimental data set used in this thesis. In general, the overall shape topologies are similar in the two populations. Of course, a more detailed representation would be based on 3D volume meshes of the entire corpus callosum, or based a series of contours form the slices forming it. This thesis is focused on single slice evaluations from the sagittal plane cross section of the data described before.

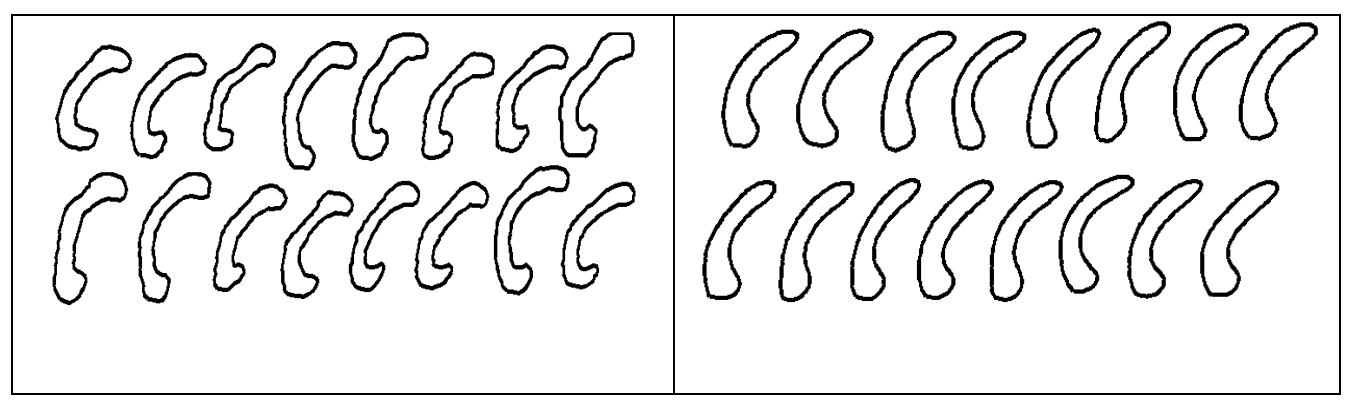

Figure 28: A sample of 16 corpus callosum contours of normal subjects and their corresponding Bezier fitting.
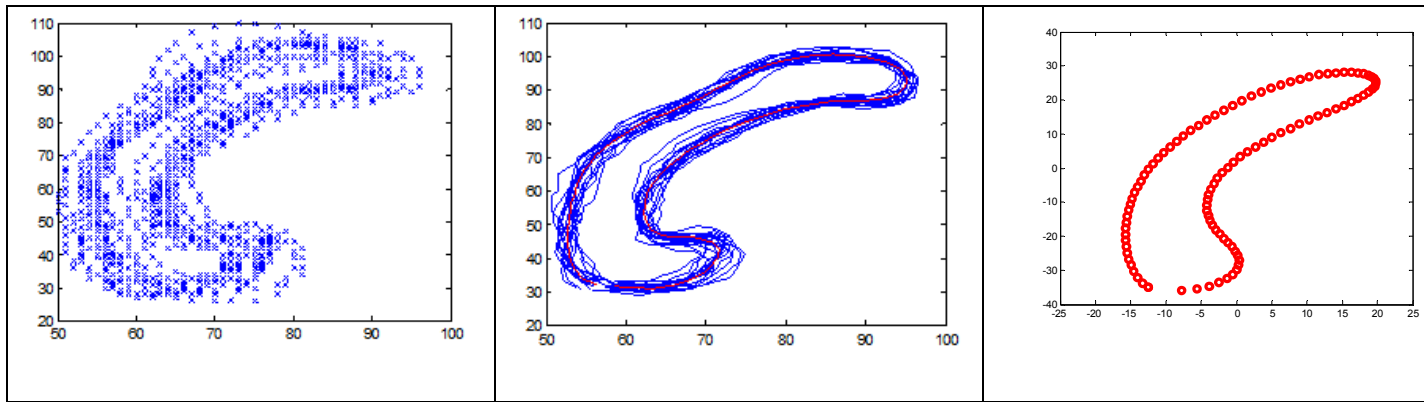

Figure 29: Individual and aligned Bezier contours for 16 normal subjects and their average shape (red). Left shows row vertices, middle show the contours and left is the mean Bezier contour.

\subsection{Classification}

\subsubsection{Feature Extraction}


Let $\left\{f_{k}\right\}_{k=1}^{N}$ be a set of $N$ features which describe the contour of a corpus colosum $\boldsymbol{C}$, where $f_{k}$ can be one of the following: (1) the coordinates of the contour control points $\boldsymbol{C}=\left[x_{1}, x_{2}, \ldots, x_{M}, y_{1}, y_{2}, \ldots, y_{M}\right]^{T}$, (2) the coordinates of the reconstructed Bezier curve $\boldsymbol{c}=\left[\hat{x}_{1}, \hat{x}_{2}, \ldots, \hat{x}_{M^{r}}, \hat{y}_{1}, \hat{y}_{2}, \ldots, \hat{y}_{M^{\prime}}\right]^{T}$ or (3) the coefficients of the fitted Bezier curve $c=\left[c_{1}, c_{2}, \ldots, c_{M}\right]^{T}$.

Let $\mathbf{A}-\left[\boldsymbol{c}_{1}^{\mathrm{A}}, \boldsymbol{c}_{2}^{\mathrm{A}}, \ldots, \boldsymbol{c}_{A}^{\mathrm{A}}\right]$ be an $N \times A$ matrix for a training set with $A$ autistic corpus colosums, and $\mathbf{C}=\left[\mathcal{c}_{1}^{\mathrm{L}}, \boldsymbol{c}_{2}^{\mathrm{r}}, \ldots, \mathcal{C}_{C}^{\mathrm{l}}\right]$ be an $N \times C$ matrix for a training set with $C$ normal corpus colosums. The average autistic and normal corpus collosum are defined as; $\overline{\mathcal{C}_{A}}=\frac{1}{A} \sum_{a=1}^{A} c_{a}^{A}$ and $\overline{\boldsymbol{C}_{C}}=\frac{1}{C} \sum_{c=1}^{C} \boldsymbol{c}_{o}^{C}$ respectively.

\subsubsection{PCA Modeling}

The zero-mean autistic and normal matrices can then computed as $\mathbf{A}^{\prime}=\left[\mathcal{C}_{1}^{\mathrm{A}}-\overline{\boldsymbol{C}_{A}}, \mathcal{C}_{2}^{\mathrm{A}}-\overline{\mathcal{C}_{A}}, \ldots, \mathcal{C}_{A}^{\mathrm{A}}-\overline{\mathcal{C}_{A}}\right] \quad$ and $\quad \mathbf{c}^{\prime}=\left[\mathcal{C}_{1}^{\mathrm{C}}-\overline{\mathcal{C}_{C^{\prime}}} \mathcal{C}_{2}^{\mathrm{C}}-\overline{\mathcal{C}_{A}}, \ldots, \mathcal{C}_{C}^{\mathrm{C}}-\overline{\mathcal{C}_{c}}\right]$ respectively. The $N \times N$ covariance matrices $\mathbf{\Sigma}_{A}$ and $\mathbf{\Sigma}_{C}$ of the autistic and normal classes can be constructed as $\mathbf{\Sigma}_{\boldsymbol{A}}=\mathbf{A}^{\prime} \boldsymbol{A}^{\prime T}$ and $\mathbf{\Sigma}_{C}=\mathbf{C}^{\prime} \mathbf{C}^{\prime T}$. The eigen-configurations are defined as the eigen vectors of the covariance matrices $\boldsymbol{\Sigma}_{A}$ and $\mathbf{\Sigma}_{E}$. Let $\mathbf{E}_{\Lambda}$ and $\mathbf{E}_{\mathbf{c}}$ be the $N \times \hat{N}$ be the most significant eigen-configurations which maintain $98 \%$ of data variability (i.e. with the largest eigen values). Thus an autistic corpus callosum $\boldsymbol{C}$ can be re-expressed as $\overline{\boldsymbol{e}}=\mathbf{E}_{\mathbf{A}}{ }^{T}\left(\boldsymbol{c}-\overline{\boldsymbol{c}_{\boldsymbol{A}}}\right)=\left[f_{1}, f_{2}, \ldots, f_{\hat{N}}\right]^{T}$ with $\hat{N} \leq N$, similarly a normal corpus callosum $\boldsymbol{e}$ can be re-expressed as $\hat{\boldsymbol{c}}=\mathbf{E}_{\mathrm{c}}{ }^{T}\left(\boldsymbol{c}-\overline{\boldsymbol{c}_{\boldsymbol{c}}}\right)=\left[\hat{f}_{1}, \hat{f}_{2}, \ldots, \hat{f}_{\mathrm{N}}\right]^{T}$. See Figs. 30 and 31 for illustrations of the process. 

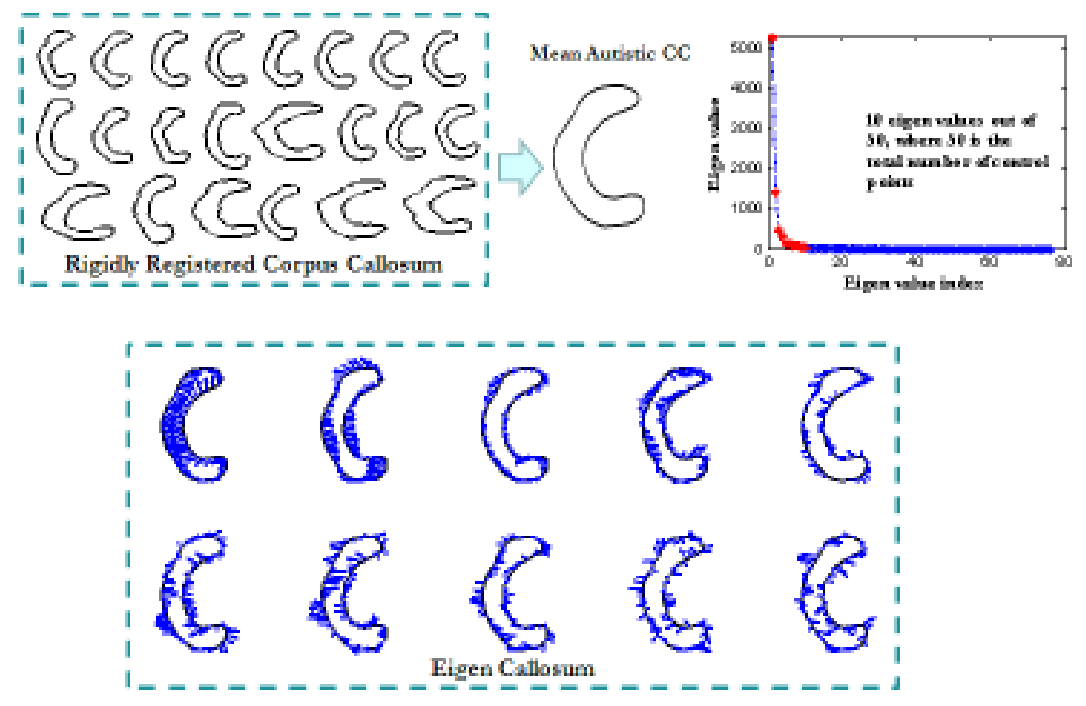

Figure 30: Given a set of training autistic corpus callosum rigidly registered, a mean autistic CC is computed. The eigen-values spectrum of the covariance matrix of the zeromean autistic CC is shown where only 10 of them maintain $98 \%$ variability of the training data, eigen callosum which are the most significant eigen vectors of the covariance matrix are plotted (as directions of variations in xy-plane) with the mean autistic CC superimposed for visualization.

Classification involves measuring the degree of similarity of a test or unknown corpus callosum and trained models of autistic and normal subjects; these models can be (1) Fourier descriptors of the mean shapes, (2) Fourier descriptors of the contour points (control or reconstructed points), (3) eigen-configurations of the contour points (control or reconstructed points) or (4) eigen-configurations of the Bezier coefficients.

We should point out the circumference of the corpus callosum of the two populations was quite distinct as shown in Table 1. In fact, for the set of data we used in this thesis, $100 \%$ classification rate was obtained based on using the circumferences. This was consistent whether we used the original contours or the Bezier curves. In practice, we may not always have non-intersecting contours of the corpus callosum and a single 
feature classification based on the circumference alone may not be robust. This also could have added as a feature to the feature vector before using the PCA

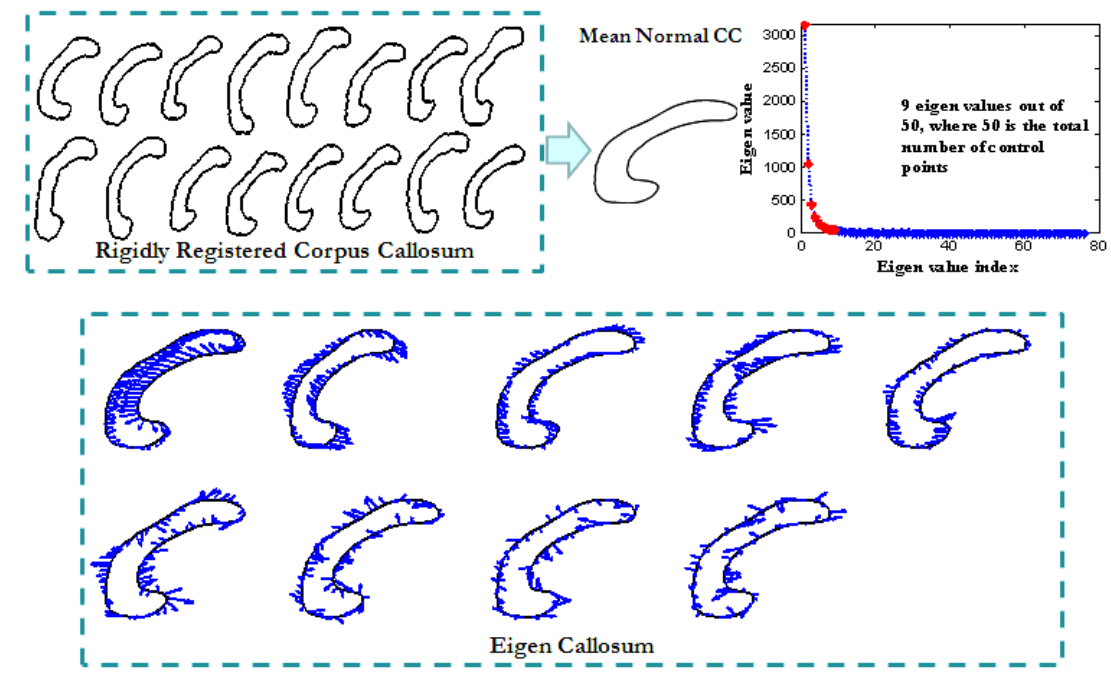

Figure 31: Given a set of training normal corpus callosum rigidly registered, a mean normal CC is computed. The eigen-values spectrum of the covariance matrix of the zeromean normal CC is shown where only 9 of them maintain 98\% variability of the training data, eigen callosum which are the most significant eigen vectors of the covariance matrix are plotted (as directions of variations in xy-plane) with the mean normal CC superimposed for visualization.

\subsubsection{Distance classification}

The L2 norm is used such that:

$$
d_{L_{z}}\left(\mathbf{f}_{i}, \mathbf{f}_{j}^{A}\right)=\left(\sum_{k=1}^{\mathbb{N}}\left|f_{i k}-f_{j k}^{A}\right|^{2}\right)^{1 / 2} \text { and } d_{L_{z}}\left(\mathbf{f}_{i}, \mathbf{f}_{j}^{C}\right)=\left(\sum_{k=1}^{N}\left|f_{i k}-f_{j k}^{C}\right|^{2}\right)^{1 / 2}
$$

Hence, $\mathbf{f}_{i} \in \mathbf{A}$ iff $d_{L_{2}}\left(\mathbf{f}_{i}, \mathbf{f}_{j}^{A}\right)>d_{L_{2}}\left(\mathbf{f}_{i}, \mathbf{f}_{j}^{C}\right)$.

\subsubsection{Bayes classification}

Bayes' classifier is also used which can be described as follows. We can express variability in the probabilistic between autistic and normal classes as follows; consider corpus Callosum $\boldsymbol{E}$ to be a continuous random variable whose distribution depends on the 
state of nature $w-\left\{w_{A}, w_{C}\right\}$ and is expressed as $p(\boldsymbol{C} \mid w)$, this is called the class (state)conditional probability density function, i.e. the probability density function for $\mathcal{C}$ given that the state of nature is $w$. Therefore the difference between $p\left(x \mid w_{A}\right)$ and $p\left(x \mid w_{C}\right)$ describes the difference in features between autistic and normal classes. The joint probability density of finding a pattern that is in category $w$ and has feature value $\boldsymbol{C}$ can be written as follows: $P(w, x)=P(w \mid x) p(x)=p(x \mid w) P(w)$, where $I(w)$ is the a priori probability, $P(w \mid x)$ is the posterior probability, i.e. the probability of the state of nature being $w=\left\{w_{A}, w_{c}\right\}$ given that the feature value $x$ has been measured, $p(x \mid w)$ is the likelihood of $w$ with respect to $x$, and $p(x)$ is the evidence factor that can merely be viewed as a scale factor that guarantees that the posterior probabilities sum to one. Therefore, the Bayesian decision rule for minimizing the probability of error becomes as follows: Decide $w_{A}$ if $P\left(w_{A} \mid \mathcal{C}\right)>P\left(w_{c} \mid \mathcal{C}\right)$ otherwise decide $w_{C}$. Equivalently; Decide $w_{A}$ if $p\left(\mathcal{C} \mid w_{A}\right) P\left(w_{A}\right)>p\left(\mathcal{C} \mid w_{C}\right) P\left(w_{\digamma}\right)$, otherwise decide $w_{\Gamma}$.

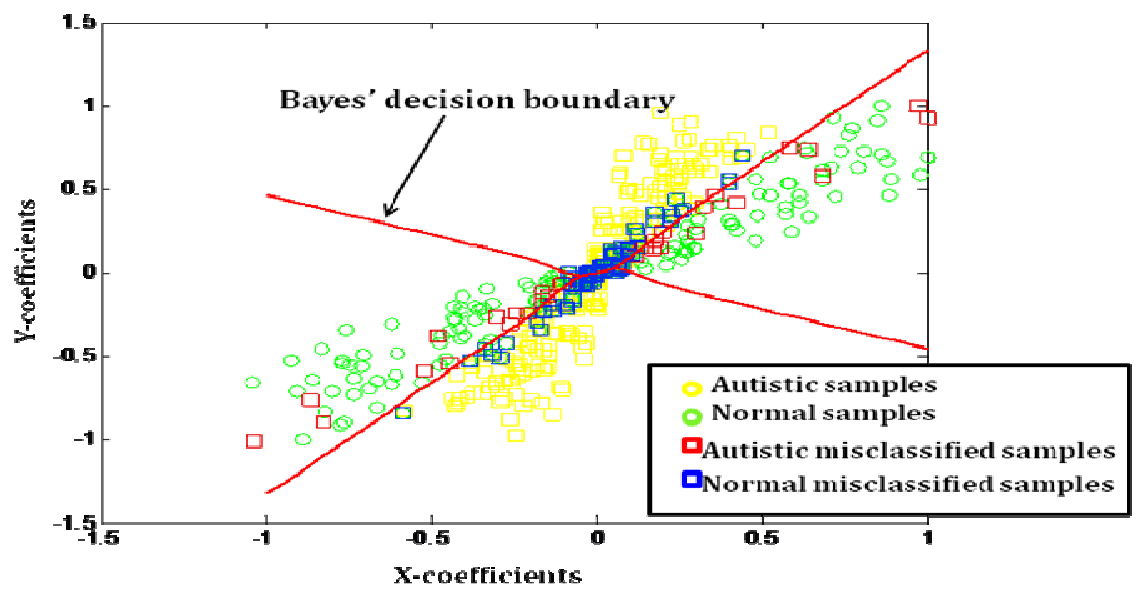


Figure 32: Bayes' classification applied on Bezier curve coefficients, the quadratic decision boundary is shown in red.

\subsubsection{The overall classification approach}

The overall process is summarized in Figure 33 below which describes the steps used for automatic classification on normal vs. autistic subjects using shape analysis of the corpus callosum. Given the sagittal MRI slice of a human's brain, the corpus callosum is segmented, then the contour is extracted via canny edge detection, this contour is then sampled to obtain the control points which are used to fit a Bezier curve after being rigidly registered to a reference corpus callosum. The Bezier curve points are then reconstructed by sampling the parameter $t$. Throughout this process we obtained three types of features which describe a corpus callosum (1) the control points, (2) the Bezier curve coefficients and (3) the reconstructed points. These features are then modeled using (a) PCA (b) Fourier descriptor and (c) probability density function (pdf). Minimum distance classifier is used for PCA-based and Fourier-based features while Bayes' classifier constructed using the trained classes pdf.

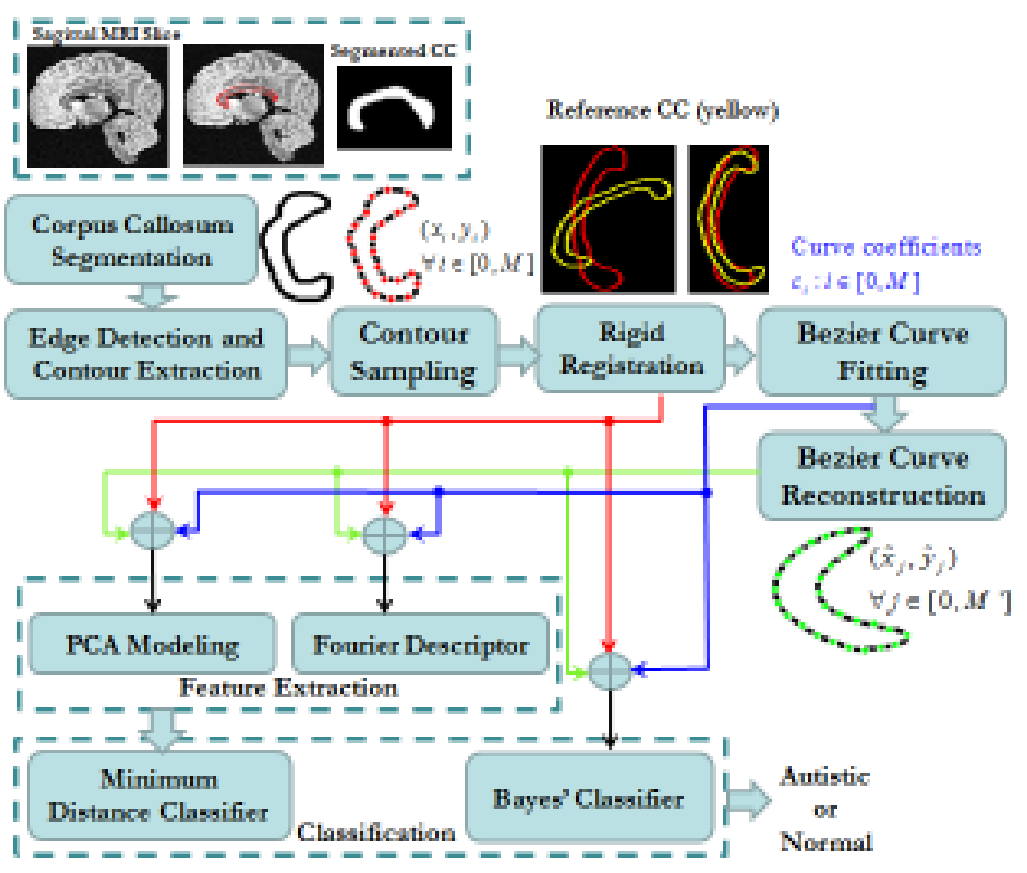

Figure 33: Automatic classification of normal and autistic subjects from shape analysis of the corpus callosum (CC). The CC contours are obtained from T1-weigheted MRI 
scans and represented by Bezier polynomials or using the Fourier Descriptors. A set of features is extracted and are used for classification.

\subsection{Results}

The following Table lists the results of the classifiers for different percentages of training and testing data from the 22 autistics and 16 normal contours. In terms of training percentages, it can be observed that the more data we have in our training set, the better results we achieve whether in autistic or normal cases. F or example, the Bayes' classifier has zero success rate in case of autistic class when we include only $25 \%$ of the autistic $\mathrm{CC}$ in the training stage, while this percentages shoots to $100 \%$ percentage when we increase the training data to $50 \%$.

Table 1: Performance of various classifiers for discrimination of normal and autistic subjects based on shape analysis of the corpus callosum contours

\begin{tabular}{|c|c|c|c|c|c|c|c|}
\hline & \multirow[b]{2}{*}{$\begin{array}{c}\text { Training } \\
\text { Percentages }\end{array}$} & \multicolumn{3}{|c|}{ Bayes' Classifier } & & & \\
\hline & & $\begin{array}{l}\text { Bezier } \\
\text { Coefficients }\end{array}$ & $\begin{array}{l}\text { Control } \\
\text { Points }\end{array}$ & $\begin{array}{l}\text { Reconstructed } \\
\text { points }\end{array}$ & & & \\
\hline \multirow{3}{*}{$\begin{array}{l}\text { Autistic } \\
\text { Success } \\
\text { Rate }\end{array}$} & $25 \%$ & $0 \%$ & $0 \%$ & $0 \%$ & & & \\
\hline & $50 \%$ & $100 \%$ & $0 \%$ & $0 \%$ & & & \\
\hline & $75 \%$ & $100 \%$ & $0 \%$ & $0 \%$ & & & \\
\hline \multirow{5}{*}{$\begin{array}{l}\text { Normal } \\
\text { Success } \\
\text { Rate }\end{array}$} & $25 \%$ & $0 \%$ & $68.75 \%$ & $100 \%$ & & & \\
\hline & $50 \%$ & $100 \%$ & $81.125 \%$ & $100 \%$ & & & \\
\hline & $75 \%$ & $100 \%$ & $93.75 \%$ & $100 \%$ & & & \\
\hline & & \multicolumn{3}{|c|}{ Fourier Descriptor } & \multicolumn{3}{|c|}{ PCA } \\
\hline & $\begin{array}{c}\text { Training } \\
\text { Percentages }\end{array}$ & $\begin{array}{l}\text { Bezier } \\
\text { Coefficients }\end{array}$ & $\begin{array}{l}\text { Control } \\
\text { Points }\end{array}$ & $\begin{array}{l}\text { Reconstructed } \\
\text { points }\end{array}$ & $\begin{array}{l}\text { Bezier } \\
\text { Coefficients }\end{array}$ & $\begin{array}{l}\text { Control } \\
\text { Points }\end{array}$ & $\begin{array}{l}\text { Reconstructed } \\
\text { points }\end{array}$ \\
\hline \multirow{3}{*}{$\begin{array}{l}\text { Autistic } \\
\text { Success } \\
\text { Rate }\end{array}$} & $25 \%$ & $95.45 \%$ & $68.2 \%$ & $100 \%$ & $82.35 \%$ & $75 \%$ & $100 \%$ \\
\hline & $50 \%$ & $86.36 \%$ & $68.2 \%$ & $100 \%$ & $100 \%$ & $100 \%$ & $100 \%$ \\
\hline & $75 \%$ & $77.27 \%$ & $90.9 \%$ & $100 \%$ & $100 \%$ & $100 \%$ & $100 \%$ \\
\hline
\end{tabular}




\begin{tabular}{|l|l|l|l|l|l|l|l|}
\hline \multirow{2}{*}{$\begin{array}{l}\text { Normal } \\
\text { Success }\end{array}$} & $25 \%$ & $12.5 \%$ & $81.25 \%$ & $0 \%$ & $84.6 \%$ & $100 \%$ & $100 \%$ \\
\cline { 2 - 8 } Rate & $50 \%$ & $31.25 \%$ & $81.25 \%$ & $0 \%$ & $100 \%$ & $100 \%$ & $100 \%$ \\
\cline { 2 - 8 } & $75 \%$ & $43.75 \%$ & $93.75 \%$ & $87.5 \%$ & $75 \%$ & $100 \%$ & $100 \%$ \\
\hline
\end{tabular}

It can be inferred from the table that the classification results of the reconstructed

Bezier points after PCA modeling outperform other features for all training percentages, and this is consistent in case of the normal or the autistic class. on the other hand, the PCA modeling of the control points maintain similar performance however this is violated in case of $25 \%$ autistic training. Fourier descriptor behaves differently, where the control points perform better for the normal class while the reconstructed points perform better for the autistic class.

Finally Bezier coefficients provide a good performance for both classes when statistically modeled and used within the framework of a Bayes' classifier.

\subsection{Summary}

This chapter studied classification of autistic vs. normal subjects using shape analysis of the contours of the corpus callosum. The Bezier Curves as well the Fourier Descriptors were tested on real data of 22 autistic and 16 normal subjects. Both approaches provide a parametric fitting of the contours, thus enabling various modeling applications. We employed various classification approaches based on the features that hold the greatest discriminatory abilities. Our investigation shows the effectiveness of the Bezier curve representation for classification. Results also showed features based on the circumference of the contours, the Bezier curve coefficients and the Fourier Descriptors possess discrimination power to distinguish autistic and normal subjects. 


\section{CHAPTER 5}

\section{SUMMARY AND POSSIBLE EXTENSIONS}

\subsection{Summary}

This thesis dealt with shape analysis of the contours of the corpus callosum segmented from T1-weighted MRI data of autistic and normal subjects. The purpose of this research is to extract features from the MRI images to enable automatic discrimination of these two populations. The ability to perform this task is extremely important for early diagnosis of autism and in follow up of the effectiveness of therapy. The literature is quite rich in neuro-imaging research as applied to autism. To date there is no automatic approach to accomplish this task using neuro-imaging analysis. The premise of the thesis has been to validate what has been known to be factual about autism using robust features that can be the basis of automatic classification techniques. From this point of view, shape analysis approaches have been implemented and tested on real data. We highlight the major accomplishments below:

- Parametric Models of the Corpus Callosum Contours

Chapter 2 described the basic techniques used in image segmentation and registration. In particular, we focused on the level set methods and its implementation by Farag and Abdelmunim 2004 [37]. We used this algorithm to segment the corpus callosum and highlight the contours using edge detection methods. Intensity based registration using the minimum square error (MSE) distance, normalized correlation and 
the mutual information techniques may be used to perform slice by slice registration of imaging data.

The points in the contours resemble vertices of a polygon on which various shape analysis methods were implemented in Chapter 3. To study the interrelationship between populations, shape registration methods were studied in chapter 2 with a focus on the variational approach of Abdelmunim and Farag, 2007 [37], the ICP approach [50] and Procrustes technique [52].

Parametric shape models were studied in Chapter 3 with focus on the Bezier Curve and Fourier Descriptors methods. Both techniques enable continuous fitting of the corpus callosum contours, and the coefficient of the fitting may be used for classification.

- Feature Extraction for Classification

Chapter 3 and chapter 4 examined the ability of the Bezier Curve and the Fourier Descriptors methods to distinguish between autistic and normal subjects. The contours of the two populations were compared with respect to the mean curves for each class. Various classification methods have been devised based on the statistics of the coefficients, the circumference of the contours and the $3 \mathrm{~dB}$ number of descriptors. In all, quite a large number of features may be extracted from these contours and compared with respect to the average of each population.

- Validation on real data with known ground truth

Chapter 4 tested the shape analysis methods on 22 autistic and 16 normal subjects. We show that the circumference of the contours and the Fourier Descriptors up to the $3 \mathrm{~dB}$ bandwidth lead to robust classification of the two populations. 
$\rightarrow$ From the computational point of view, a complete system has been developed on Matlab which takes an MRI scan, segment the corpus callosum, outline its contour, co-register with respect to the average shape and make a decision with respect to similarity.

$\rightarrow$ From a clinical point of view, we have developed simple metrics for classification that are based on solid mathematical foundation. The accuracy of these measures on a sample contour of autistics and normal subjects holds great promise. The availability of more clinical data, specially, high resolution MRI scans on 3T scanners would provide a much needed row data for testing and validating the classifiers.

$\rightarrow$ From a learning point of view, considerable experience has been gained in numerical approaches for biomedical imaging analysis. Working on a thesis project has cause me to think out problems in a much more connected forum of the various parts of my education than I ever experienced by just taking individual classes. The research has also been a direct application of the tools that I learned from ECE 643 “Biomedical Computing”, Spring 2009 and ECE 600 "Shape Analysis", Summer 2009.

\subsection{Possible Extensions}

The extensions to this work are immediate and would include the following: 
1. Using 3D data instead of single contours. Shape analysis may be performed as average of a number of contours (e.g., from the slices of the Corpus Callosum taken at a particular scanning plane).

2. Use of more clinical data, especially high resolution $3 \mathrm{~T}$ scans of autistic and normal subjects.

3. Explore further the features that may be described from the Bezier Curves, and consider a decision fusion algorithm based on these curves and the Fourier Descriptors and test the algorithms using a larger number of control points.

These ideas will be explored with Dr. Casanova and Dr. Farag at the CVIP Lab in order to devise the best approaches to explore further research on this very important subject. 


\section{REFERENCES}

1. N.J. Minshew, J.B. Payton, "New perspectives in autism", Part I. The clinical spectrum of autism, Curr. Probl. Pediatr. 18 (1988), pp 561-610.

2. Yeargin-Allsop M, Rice C, Karapurkar T, Doernber N, Boyle C, Murphy C. "Prevalence of autism in a US metropolitan area". JAMA 2003;289:48 -55.

3. Tidmarsh L, Volkmar FR. "Diagnosis and epidemiology of autism spectrum disorders”, Can J Psychiatry 2003;48(8):517-25.

4. P. Bolton et al., "A case-control family history study of autism", J. Child Psychol. Psychiatry 35 (1994), pp 877-900.

5. N.J. Minshew, J.B. Payton, "New perspectives in autism", Part II. The differential diagnosis and neurobiology of autism, Curr. Probl. Pediatr. 18 (1988), pp 613694.

6. M.C. Stevens et al., "Subgroups of children with autism by cluster analysis: a longitudinal examination", J. Am. Acad. Child Adolesc. Psychiatry, 39 (2000), pp 346-352.

7. Kanner L. "Autistic disturbances of affective contact", Nervous Child 1943; 2:21250.

8. Bailey A, Luthert P, Bolton P.“Autism and megalencephaly”. Lancet 1993; 341: 1225-6.

9. Kemper TL, Bauman ML. "The contribution of neuropathologic studies to the understanding of autism". Neurol Clin 1993; 11: 175-187.

10. Davidovitch M, Patterson B, Gartside P. "Head circumference measurements in children with autism". J Child Neurol 1996; 11: 389-93.

11. Miles JH, Hadden LL, Takahashi TN, Hillman RE. "Head circumference is an independent clinical finding associated with autism”. AmJMed Genet 2000; 95: 33950 .

12. Aylward EH, Minshew NJ, Field K, Sparks BF, Singh N. "Effects of age on brain volume and head circumference in autism”. Neurology 2002; 59: 175-83.

13. Courchesne E, Carper R, Akshoomoff N. "Evidence of brain overgrowth in the first year of life in autism". J Am Med Assoc 2003; 290: 337-44. 
14. Filipek PA, Richelme C, Kennedy DN, Rademacher J, Pitcher DA, Zidel S, et al. "Morphometric analysis of the brain in developmental language disorders and autism". Ann Neurol 1992; 32: 475.

15. Courchesne E, Karns C, Davis H, Ziccardi R, Carper R, Tigue Z, and others. "Unusual brain growth patterns in early life in patients with autistic disorder: an MRI study". Neurology 2001;57:245-54.

16. Guerin P, Lyon G, Barthelemy C, Sostak E, Chevrollier V, Garreau B, et al. "Neuropathological study of a case of autistic syndrome with severe mental retardation". Dev Med Child Neurol 1996; 38: 203-11.

17. Egaas B, Courchesne E, Saitoh O. "Reduced size of corpus callosum in autism. Arch Neurol”, 1995;52:794-801.

18. Piven J, Bailey J, Ransom B, Arndt S. "An MRI study of the corpus callosum in autism. Am J Psychiatry”, 1997;154:1051-6.

19. Manes F, Piven J, Vrancic D, Nanclares V, Plebst C Starkstein S. "An MRI study of the corpus callosum and cerebellum in mentally retarded autistic individuals", $\mathrm{J}$ Neuropsychiatry Clin Neurosci 1999;11:470-4.

20. Hardan AY, Minshew NJ, Keshavan MS. "Corpus callosum size in autism", Neurology 2000;55:1033-6.

21. Christine N. Vidal, Rob Nicolson, Timothy J. DeVito, Kiralee M. Hayashi, Jennifer A. Geaga, Dick J. Drost, Peter C. Williamson, Nagalingam Rajakumar, Yihong Sui, Rebecca A. Dutton, Arthur W. Toga, and Paul M. Thompson, "Mapping Corpus Callosum Deficits in Autism: An Index of Aberrant Cortical Connectivity", The Canadian Journal of Psychiatry-In Review.

22. Cody H, Pelphrey K, Piven J. "Structural and functional magnetic resonance imaging of autism". Int J Dev Neurosci 2002;20:421-38.

23. Williams RS, Hauser SL, Purpura DP, DeLong GR, Swisher CN. "Autism and mental retardation: neuropathologic studies performed in four retarded persons with autistic behavior". Arch Neurol 1980; 37: 749-53.

24. Ritvo ER, Freeman BJ, Scheibel AB. "Lower Purkinje cell counts in the cerebella of four autistic subjects: initial findings of the UCLA-NSAC autopsy research report”. Am J Psychiatry 1986; 143: 862-6. 
25. Lee M, Martin-Ruiz C, Graham A, Court J, Jaros E, Perry R. "Nicotinic receptor abnormalities in the cerebellar cortex in autism". Brain 2002; 125: 1483-95.

26. Bailey A, Luthert P, Dean A, Harding B, Janota I, Montgomery M, et al.“A clinicopathological study of autism". Brain 1998; 121: 889-905.

27. Courchesne E, Yeung-Courchesne R, Press G, Hesselink J, Jernigan T. "Hypoplasia of cerebellar vermal lobules VI and VII in autism". N Engl J Med 1988;318:1349-54.

28. Allen G, Courchesne E. "Differential effects of developmental cerebellar abnormality on cognitive and motor functions in the cerebellum: an fMRI study of autism”. Am J Psychiatry 2003;160:262-73.

29. Barnea-Goraly N, Kwon H, Menon V, Eliez S, Lotspeich L, Reiss AL. "White matter structure in autism: Preliminary evidence from diffusion tensor imaging". Biological Psychiatry 2004; 55:323-326.

30. Cynthia Mills Schumann et al., "Magnetic Resonance Imaging of the PostMortem Autistic Brain", J. of Autism and Developmental Disorders, Vol. 31, No. 6, December 2001.

31. Hines M, Chiu I, McAdams LA, Bentler PM, Lipcamon J (1992). Cognition and the corpus callosum: verbal fluency, visuospatial ability, and language lateralization related to midsagittal surface areas of callosal subregions. Behav Neurosci 106: 3-14.

32. Ringo JL. (1991). Neuronal interconnection as a function of brain size. Brain, Behavior and Evolution 38:1-6.

33. Hossam E Abd El Munim and Aly A. Farag, "Elastic Registration of the Corpus Callosum," Proc. of IEEE International Conference on Image Processing (ICIP'07), St. Antonio, Texas, September 16-19, 2007, pp. V-377 - V-380.

34. Manuel F. Casanova, Aly Farag, Ayman EL-BAZ, Meghan Mott, Hossam Hassan, Rachid Fahmi, Andrew E. Switala, "Abnormalities of the Gyral Window in Autism: A Macroscopic Correlate to a Putative Minicolumnopathy," Journal of Special Education and Rehabilitation, pp. 1:85-101, 2007.

35. Xiaoyan Ke; Shanshan Hong; Tianyu Tang; Haiqing Huang; Bing Zou; Huiguo Li; Yueyue Hang; Zuhong Lu; Bioinformatics and Biomedical Engineering, 2008. ICBBE 2008. The 2nd International Conference on 16-18 May 2008 Page(s):2409 -2413 . 
36. http://www.nlm.nih.gov/medlineplus/ency/imagepages/8753.htm

37. Aly A. Farag and H. Hassan, Adaptive Segmentation of Multi-modal 3D Data Using Robust Level Set Techniques, Proc. of International Conference on Medical Image Computing and Computer-Assisted Intervention (MICCAI'04), Saint Malo, France, September 26-29, 2004, pp. 143-150.

38. Hossam Abdelunim and A. A. Farag, "Shape Representation and Registration using Vector Distance Functions," Proc. of IEEE Conference on Computer Vision and Pattern Recognition (CVPR'07), Minneapolis, MN, June 18-23, 2007, pp. Y1-Y8.

39. Hossam Abdelmunim, "Implicit Curve/Surface Evolution with Application to the Image Segmentation Problem", PhD Thesis, Computer Vision and Image Processing Laboratory, University of Louisville, May 2007.

40. Rachid Fahmi and Aly A. Farag "A Global-to-Local 2D Shape Registration in Implicit Spaces Using Level Sets," Proc. of IEEE International Conference on Image Processing (ICIP'07), St. Antonio, Texas, September 16-19, 2007, VI-237VI-240.

41. Rachid Fahmi, "Variational Methods For Shape and Image Registrations", PhD Thesis, Computer Vision and Image Processing Laboratory, University of Louisville, May, 2008.

42. S. Osher and J. Sethian, "Fronts propagating with curvature-dependent speed: algorithms based on Hamilton-Jacobi formulations," J. Comp. Phys., Vol. 79, pp. 12-49, 1988.

43. J. A. Sethian, Level set methods and fast marching methods, Cambridge University Press, Cambridge, UK, 1999.

44. T. Chan and L. Vese, "Active contours without edges," IEEE Transactions on Image Processing., Vol. 10, pp. 266-277, 2001.

45. Hossam Abdelmunim and A. A. Farag, "A Shape-based Segmentation Approach: An Improved Technique using Level Sets," Proc. of International Conference on Computer Vision (ICCV), Beijing, China, October 15-21, 2005, pp. 930-935.

46. Hossam Abd EL Munim and Aly A. Farag, "Curve/Surface Representation and Evolution using Vector Level Sets with Application to the Shape-based Segmentation Problem," IEEE Transactions on Pattern Analysis and Machine Intelligence - Vol. 29, No. 6, pp. 945-958, June 2007. 
47. Barbara Zitová and Jan Flusser, Image registration methods: a survey, Image and Vision Computing, Volume 21, Issue 11, October 2003, Pages 977-1000.

48. W. M. Wells, P. Viola, H. Atsumi, S. Nakajima, and R. Kikinis, "Multi-modal Volume Registration by Maximization of Mutual Information," Medical Image Analysis, vol. 1, no. 1, pp. 35-51, 1996.

49. R. O. Duda, P. E. Hart, and D. G. Stork, Pattern Classification. New York: John Wiley \& Sons, 2nd edition, 2001.

50. Paul J. Besl, Neil D. McKay: A Method for Registration of 3-D Shapes. IEEE Trans. Pattern Anal. Mach. Intell. 14(2): 239-256 (1992).

51. M. Stegmann, “Active Appearance Models: Theory, Extension and Cases,” MS Thesis, IMM DTU, 2000.

52. T. F. Cootes and C. J. Taylor, "Statistical Models of Appearance for Computer Vision," Technical Report, ISBE, University of Manchester, 2004.

53. Matlab, www.mathworks.com.

54. D. G. Kendall. The diffusion of shape. Advances in Applied Probability, 9:428 430, 1977.

55. L. Dryden and K. V. Mardia. Statistical Shape Analysis. John Wiley \& Sons, 1998.

56. Stegmann, Mikkel B., Gomez, David Delgado: A Brief Introduction to Statistical Shape Analysis, Technical, University of Denmark, Lyngby, 2002.

57. Foley, Van Dam et al. Computer Graphics, Principles and Practice. AddisonWesley, 1991.

58. A. Papoulis, the Fourier Integral and its Applications, McGraw-Hill, New York, 1962.

59. C.T. Zahn and R.Z. Roskies, "Fourier descriptors for plane close curves", IEEE Trans. Computers, Vol C-21, March 1972, pp. 269-281.

60. O. Betrand, R. Queval, H. Matre, "Shape Interpolation by Fourier Descriptors with Application to Animation Graphics", Signal Processing, June 1981, 4:53-58. 
61. Pete E. Lestrel, "Fourier Shape Descriptors and their Applications in Biology," ISBN 052145201 hardback, Published by Cambridge University Press, 1997. Chapter 2.

62. V. Solachidis and I. Pitas, "Watermarking polygonal lines using Fourier descriptors," IEEE Computer Graphics and Applications, May/June 2004.

63. Diana L. Guidry, "Active Contours: With Application to Motion Artifact Cancellation and Segmentation in MRI," Master Thesis of Electrical Engineering, August 1999.

64. Aly A. Farag, Hossam Abdelmunim, and Manuel F. Casanova, "Experiments on Classification of Autistic and Normal Subjects Using Frequency-Domain Analysis of T1-weighted MRI Data of Human Brain," Technical Report, Computer Vision and Image Processing Laboratory, University of Louisville, October 2007.

65. Hossam Abdelmunim, Aly A. Farag, and Manuel F. Casanova, "FrequencyDomain Analysis of the Human Brain for Studies of Autism," Proceedings, 7th IEEE International Symposium on Signal Processing and Information Technology, ISSPIT 2007, Cairo, Egypt, December 15-18, 2007, pp. 1198-1203. 
Ahmed A. Farag was born in West Lafayette, Indiana, USA, moved to Louisville, Kentucky in 1990, where he received all his education. He received this High School Diploma from Louisville Traditional Male High School in 2004. He joined the University of Louisville in 2004, where he received his Bachelor degree in Electrical and Computer Engineering in December 2008. He is scheduled to receive the Masters of Engineering Degree in December 2009. He is interested in robotics and biomedical research. During 2006-2008, he performed Co-Op research at the Computer Vision and Image Processing Laboratory, University of Louisville, USA, working on autonomous robotics. He has served as Vice President of the Louisville IEEE Chapter, Dell Campus Representative and worked at various Computer and Consumer Electronics outlets in Louisville. He received IEEE student award in 2009, and first place award in the E-Day Competition in 2007. Among his talents are computer design, networking, robotics and biomedical image analysis algorithms. 